\title{
Variability and periodicity of field M dwarfs revealed by multichannel monitoring
}

\author{
B. Rockenfeller, C. A. L. Bailer-Jones, and R. Mundt
}

Max-Planck-Institut für Astronomie, Königstuhl 17, 69117 Heidelberg, Germany

e-mail: calj@mpia.de

Received 5 September 2005 / Accepted 15 November 2005

\section{ABSTRACT}

We present simultaneous, multiband photometric monitoring of 19 field dwarfs covering most of the M spectral sequence (M2-M9). Significant variability was found in seven objects in at least one out of the three channels $I, R$ and $G$. Periodic variability was tested with a CLEAN power spectral analysis. Two objects, LHS370 (M5V) and 2M1707+64 (M9V), show periods of $5.9 \pm 2.0$ and $3.65 \pm 0.1 \mathrm{~h}$ respectively. On account of the agreement with the typical values of $v \sin i$ published for M dwarfs (Mohanty \& Basri 2003, ApJ, 583, 451), we claim these to be the objects' rotation periods. Three further objects show possible periods of a few hours. Comparing the variability amplitude in each channel with predictions based on the synthetic spectra of Allard et al. (2001, ApJ, 556, 357), we investigated the source of variability in LHS370 and 2M1707+64. For the latter, we find evidence for the presence of magnetically-induced cool spots at a temperature contrast of 4-8\%, with a projected surface coverage factor of less than 0.075. Moreover, we can rule out dust clouds (as represented by the COND or DUSTY models) as the cause of the variability. No conclusion can be drawn in the case of LHS370. Comparing the frequency of occurrence of variability in this and various $\mathrm{L}$ dwarf samples published over the past few years, we find that variability is more common in field $\mathrm{L}$ dwarfs than in field $\mathrm{M}$ dwarfs (for amplitudes larger than $0.005 \mathrm{mag}$ on timescales of 0.5 to $20 \mathrm{~h}$ ). Using the homogeneous data sets of this work and Bailer-Jones \& Mundt (2001, A\&A, 367, 218), we find fractions of variable objects of $0.21 \pm 0.11$ among field $\mathrm{M}$ dwarfs and $0.70 \pm 0.26$ among field $\mathrm{L}$ dwarfs (and $0.29 \pm 0.13,0.48 \pm 0.12$ respectively if we take into account a larger yet more inhomogeneous sample). This is marginally significant ( $2 \sigma$ deviation) and implies a change in the physical nature and/or extent of surface features when moving from M to L dwarfs.

Key words. methods: data analysis - techniques: photometric - stars: late-type - stars: rotation - starspots - stars: variables: general

\section{Introduction}

Photometric and spectroscopic monitoring of very low mass stars and substellar objects has been performed over the last ten years by several groups and has led to new insights into the processes occurring in these objects. Since stars on the main sequence become fully convective below a mass of about $0.3-0.4 M_{\odot}$ (roughly corresponding to a spectral type of M3 to M4), magnetic fields cannot be maintained in stars of lower mass or later type via an $\alpha \Omega$ dynamo. An alternative dynamo may come into operation, for example an $\alpha^{2}$-dynamo (Chabrier $\&$ Küker [arXiv: astro-ph/0510075]). A change in the dynamo mechanism with spectral type could directly affect the activity of $\mathrm{M}$ dwarfs as measured by $\mathrm{H}_{\alpha}$ emission, for example. Early M dwarfs in most cases do not show any detectable $\mathrm{H}_{\alpha}$ emission whereas it increases at mid and late $\mathrm{M}$ type. Furthermore, a saturation-type relation between rotation and activity has been confirmed by Mohanty \& Basri (2003) and Delfosse et al. (1998). The detection of flares on these objects also proves the existence of magnetic fields. However, magnetic activity strongly decreases again with early L type (West et al. 2004) which can be explained via an increasingly neutral photosphere and the onset of dust formation (Mohanty et al. 2002). Since photometric variability can be caused by both magnetic activity (through star spots) and dust clouds, it is not clear whether variability is more frequent or less frequent in $\mathrm{M}$ dwarfs than in L dwarfs. Measurements of $v \sin i$ values show that later-type objects on average rotate much faster and lead to expected rotation periods of about 1.5-13 h (Bailer-Jones 2004) for L dwarfs, whereas $M$ dwarfs can have significantly longer periods of up to two days. Thus it appears that the spindown timescale is much longer for later-type objects.

Stellar/substellar rotation periods can be measured directly from photometric time series if the objects show surface brightness inhomogeneities. This also permits a study of multiperiodicity and/or nonperiodic variability. Candidates for the surface features are magnetically induced spots and, because of their lower effective temperature, dust clouds for late $\mathrm{M}$ and $\mathrm{L}$ dwarfs. By modelling the variability at different wavelengths, we can attempt to infer the physical properties of the atmosphere and surface features in individual cases. The few attempts to attribute a specific physical cause of the variability (via time-resolved spectroscopy) have been rather inconclusive (Bailer-Jones 2002; Clarke et al. 2003, 
Bailer-Jones \& Lamm 2003), however, partly due to the high multifrequency photometric sensitivity ( $1 \%$ or better) required to distinguish between mechanisms.

There have been several one- or two-band photometric surveys of ultra cool dwarfs (UCDs) of both young cluster objects and older field objects, mostly in the $I$-band but also in the $R$ or near infrared bands. A comparison of these reveals variability (periodic or nonperiodic) to be present in about $40 \%$ of objects in each of these two age groups (Bailer-Jones 2005). Interestingly, periodic variability is more common among the younger cluster sample, whereas cases of nonperiodic variability are more frequent in the older field sample. This could, in principle, be either an age or a spectral type effect. An age effect might be related to a decline in activity, possibly from the dissipation of disks, although we have no explicit mechanism for this. A spectral type effect could be related to the onset of dust formation and, specifically, the dynamics of large scale dust clouds: Bailer-Jones \& Mundt (2001, henceforth BJM01) proposed a "masking hypothesis" in which the rapid formation and evolution of dust clouds masks the rotational modulation of the light curves of $\mathrm{L}$ dwarfs, thus accounting for their nonperiodic variability (also see Bailer-Jones 2004). We cannot distinguish between these two effects in the samples to date, because there is a broad age-spectral type (SpT) correlation in the object selection: most $\mathrm{M}$ dwarfs monitored were in young clusters (1-100 Myr) whereas the L dwarfs were field objects (probably more than several hundred Myr in age). Specifically, in our earlier work (BJM01) - where we monitored 21 M6-L5 dwarfs - we found variablity to be more common for objects later than M9. However, as stated in that paper, because of a coarse age-SpT correlation in the sample, we could not distinguish between an age and a SpT dependence based only on those data.

The main objective of the present work is to remove this correlation by extending the survey of BJM01 to (older) field $\mathrm{M}$ dwarfs. This represents a control sample against which the occurrence, periods, amplitudes and nature of variability in the field L dwarf sample of BJM01 can be compared, i.e. two populations with similar ages. Early and mid M dwarfs do not show photospheric dust formation (either theoretically or observationally), so together with the L dwarf samples allows us to study the link between variability and dust. In addition to significantly increasing the time-resolved data on field $\mathrm{M}$ dwarfs in $G, R$ and $I$, we are also able to constrain the physical cause of the variability in individual cases.

The structure of the paper is as follows: the next section describes the target selection and data acquisition process. In Sect. 3 we present the basic data reduction steps, photometry and a reliable error description model which together allow us to investigate low-amplitude variability. Methods for analysing general and periodic variability are discussed in Sect. 4; here we also describe our Monte Carlo approach to estimating the reliability and uncertainty in the period detection process. We briefly mention the multichannel detection of a massive flare in an M9 dwarf. This is reported in more detail in Rockenfeller et al. (2006). Section 5 deals with the overall results plus details on some individual targets. Two of these objects are studied for the cause of the observed variability and a comparison of the frequency of variability for $\mathrm{L}$ and $\mathrm{M}$ dwarfs is performed.
We finish with the conclusions in Sect. 6. For a more detailed description and discussion of the methods and for comments on every individual target see Rockenfeller (2005).

\section{Data acquisition}

\subsection{Target selection}

The target list was assembled by selecting bright $\mathrm{M}$ dwarfs $\left(m_{I}<17.0 \mathrm{mag}\right)$ which are visible from the Calar Alto observatory, Spain, in June for several hours at an airmass of less than 2.0. Many of the objects are bright enough such that the $G$-band data have a relatively high signal-to-noise ratio (SNR). Strong activity as measured by $\mathrm{H}_{\alpha}$ was avoided and they were chosen to cover most of the spectral M-type sequence (M2 to M9). A total of 19 field M-dwarfs were observed over the two observing seasons. Target details are given in Table 1.

\subsection{Observations}

To perform simultaneous multiband photometry, we used the BUSCA four channel CCD camera at the $2.2 \mathrm{~m}$ telescope at Calar Alto Observatory, Spain. This instrument uses dichroics to split the light beam into four wavelength bands, namely UV, $G, R$ and $I$. The UV, $G$ and $R$ passbands are defined by the CCD response and dichroic transmission function; for the $I$ band we additionally used a Bessel $I$ filter (see Fig. 1). Each channel is equipped with a CCD485 Lockheed Martin 4K × 4K CCD. The CCDs are thick, except for the UV channel which uses a backside-illuminated thinned chip. For all CCDs $2 \times 2$ binning was used, reducing read-out time and leading to a pixel scale of $0.352^{\prime \prime}$. The field-of-view of $12^{\prime} \times 12^{\prime}$ allowed us to select a large number of reference stars for differential photometry. For use throughout this paper, we define an adjusted Julian day (AJD) by AJD = JD - 2450000 . Observations were conducted in two runs: the first with 8 usable nights in June 2002 (AJD 2424.4-2431.7) and the second with 9 usable nights in June 2003 (AJD 2794.4-2803.6). On each night, two or three targets were observed alternately and for each target, data were collected on two or three nights. Integration times of 45 to $300 \mathrm{~s}$ were used depending on target brightness and weather conditions, to achieve high signal-to-noise data and still retain sensitivity to periods in the sub-hour regime.

\section{Data reduction}

\subsection{Basic reduction steps}

All science images were reduced using the $\operatorname{IRAF}^{1}$ package. $U V$-band data could not be used due to the faintness of the $M$ dwarfs in this spectral range, other than for finding flares in the targets' time series (see Sect. 4.3). We reduced the other three channels with the same reduction steps, with parameters set to achieve homogeneous, comparable photometry. The basic reduction steps include an overscan subtraction and flat fielding process. Because non-thinned CCDs were used, no

1 The Image Reduction and Analysis Facility, provided by the National Optical Astronomy Observatories (NOAO). 
Table 1. Target properties: full name, spectral type, I magnitude, number of images in total, year of observation, nights of observations, duration of the observations on the individual nights, additional information and the references to these. Objects are sorted by increasing right-ascension.

\begin{tabular}{|c|c|c|c|c|c|c|c|c|}
\hline Target & SpT & $m_{I}$ & $N_{\text {obs }}$ & Year & Nights & Duration $[\mathrm{h}]$ & Further information & Reference \\
\hline 2MASSW J1311391+803222 & M8 & 15.9 & 33 & 2002 & 5,8 & $5.9,6.7$ & $\mathrm{H}_{\alpha}=3.0$ & G00 \\
\hline 2MASSW J1336504+475131 & M7 & 15 & 66 & 2003 & 9,10 & $4.6,4.8$ & $v \cdot \sin i=30, \mathrm{H}_{\alpha}=3.2,5.0$ & G00, R02 \\
\hline 2MASSW J1344582+771551 & M7 & 15.2 & 41 & 2002 & 1,4 & $5.2,6.2$ & $\mathrm{H}_{\alpha}=2.7$ & G00 \\
\hline LHS370 & M5 & 12.3 & 20 & 2002 & 8 & 6.1 & - & - \\
\hline LHS2930 & M6.5 & 13.3 & 58 & 2003 & 7,8 & $7.1,7.0$ & $R / R_{\text {Sun }} \approx 0.33, T_{\text {eff }}=2687 \pm 65 \mathrm{~K}$ & H04, D02 \\
\hline CTI153948.1+280322 & M2 & 14.9 & 50 & 2003 & 1,3 & $4.9,6.9$ & Binary Star; $v \sin i=7.0, \mathrm{H}_{\alpha}=0.7$ & M03 \\
\hline 2MASSW J1546054+374946 & M7.5 & 15.2 & 51 & 2003 & $4,5,6$ & $6.4,2.6,2.4$ & $\mathrm{H}_{\alpha}=10.9$ & $\mathrm{G} 00, \mathrm{C} 03$ \\
\hline LHS3189 & M5.5 & 14.9 & 49 & 2002 & 1,2 & $6.5,6.7$ & - & B91 \\
\hline 2MASS J16272794+8105075 & M9 & 16.4 & 52 & 2003 & $4,5,6$ & $6.4,2.9,2.4$ & & G00 \\
\hline CTI162920.5+280239 & M4 & 15.6 & 49 & 2003 & 1,3 & $5.2,6.4$ & $\mathrm{H}_{\alpha}=10.9$ & - \\
\hline 2MASSW J1707183+643933 & M9 & 15.9 & 34 & 2002 & 4,5 & $6.4,5.6$ & $\begin{array}{l}T_{\mathrm{eff}} \approx 2350 \mathrm{~K}, \mathrm{H}_{\alpha}=9.8, \\
\log g=5.16, M / M_{\text {Sun }}=0.062\end{array}$ & G00, G03 \\
\hline CTI170958.5+275905 & M5.5 & 13.9 & 54 & 2003 & $4,5,6$ & $6.7,2.8,2.3$ & & K94 \\
\hline 2MASSW J1714523+301941 & M6.5 & 14.9 & 51 & 2003 & 1,3 & $6.1,5.4$ & $\begin{array}{l}T_{\mathrm{eff}} \approx 2775 \mathrm{~K}, v \cdot \sin i<4, \\
\mathrm{H}_{\alpha}=3.2,5.4\end{array}$ & G00, R02 \\
\hline LHS3307 & M5 & 15 & 57 & 2003 & 9,10 & $6.8,4.1$ & - & B91 \\
\hline 2MASS J17501291+4424043 & M7.5 & 15.6 & 64 & 2003 & 7,8 & $6.8,6.9$ & $\mathrm{H}_{\alpha}=2.7$ & G00 \\
\hline LHS3339 & M6 & 14.0 & 50 & 2002 & 1,2 & $6.0,6.4$ & $T_{\mathrm{eff}}=2957 \pm 70 \mathrm{~K}, R / R_{\text {Sun }} \approx 0.33$ & L00, D02 \\
\hline 2MASSW J1757154+704201 & M7.5 & 14.2 & 38 & 2002 & 4,5 & $6.3,7.0$ & $\mathrm{H}_{\alpha}=3.0$ & G00 \\
\hline CTI180120.1+280410 & M2.5 & 14.8 & 57 & 2003 & 7,8 & $6.2,6.9$ & - & - \\
\hline LHS3376 & M4 & 10.7 & 30 & 2002 & 8 & 6.0 & $T_{\mathrm{eff}} \approx 3100 \mathrm{~K}, v \cdot \sin i=14.6 \pm 1.0$ & D98 \\
\hline
\end{tabular}

B91: Bessell (1991); K94: Kirkpatrick et al. (1994); D98: Delfosse et al. (1998); G00: Gizis et al. (2000).

L00: Leggett et al. (2000); R02: Reid et al. (2002); D02: Dahn et al. (2002).

M03: Mohanty \& Basri (2003); C03: Cruz et al. (2003); G03: Gorlova et al. (2003); H04: Henry et al. (2004).

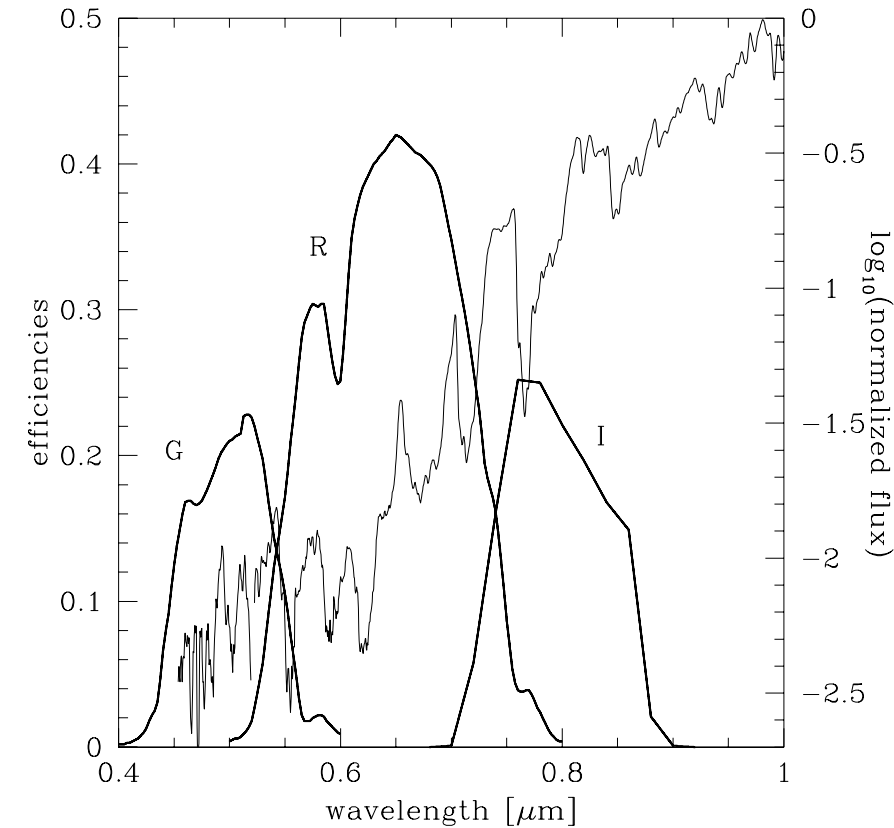

Fig. 1. The total efficiencies of the BUSCA $G, R$ and $I$ channels, taking into account the dichroics, the CCD efficency and the Bessell- $I$ filter used in the $I$-band. Also plotted is the spectrum of the M4.5 dwarf DENIS P-J1158-1201 of Martín et al. (1999).

fringes can be seen in any of the images and hence fringe correction was not performed.

The zero integration time frames, which were obtained to correct for two-dimensional bias patterns in the science images, are useless: they all show vertical bars caused by the read-out electronics which do not appear in the science frames, a known problem that occurs frequently when using BUSCA. To partly compensate for this, a first-order fit to the overscan area was subtracted from each science and flat field frame. This corrects at least for one-dimensional patterns in the y-direction of the bias level. Because residual two-dimensional patterns are only weak and do not show a strong gradient on the scale of the applied sky apertures, their contribution to the total photometric error is well below $0.5 \%$. We used an clipped average of ten dome flat fields to correct for changes in the pixels' quantum efficiencies. Individual flat field frames were applied separately for each channel and each night.

\subsection{Photometry}

To achieve the desired accuracy of better than $0.01 \mathrm{mag}$, differential photometry was performed to reduce the effects of temporal variations in Earth's atmosphere. Aperture photometry was done on the target and tens of reference stars; these were chosen according to the criteria used in BJM01. Refer also to that paper for a description of differential photometry. Although various aperture radii were tested, the results we show are with a radius of 6 pixels (except for LHS370, see Sect. 5.2) which we found to maximize the signal-to-noise ratio (SNR). The large field-of-view guarantees that in most cases more than 15 (and even up to 50) non-variable reference stars could be found within the target field, ensuring a stable reference flux. For the procedure of creating the reference star list, see the next section. We excluded the presence of 
significant second-order-extinction (SOE; see Bailer-Jones \& Lamm (2003) for a discussion) in our data by: plotting relative magnitudes versus airmass; comparing the relative light curves formed with only blue and only red reference stars; plotting the variability measure of all reference stars of a field ( $\chi^{2}$ values, see Sect. 4.1) versus the $R-I$ colour. See Rockenfeller (2005) for more details.

\subsection{Error sources and estimation}

An accurate error estimate is important for assessing the presence of variability. As discussed in Bailer-Jones \& Mundt (1999), the total photometric error is composed of formal (Poisson noise) and informal error contributions. As imperfect flat fielding is thought to dominate the informal error, we first tried to model the total photometric error $\left(\sigma_{\text {tot }}\right)$ by adding a constant term (of $0.5 \%$ ) in quadrature to the theoretical (formal) errors provided by IRAF $\left(\sigma_{\mathrm{IRAF}}\right)$. However, this lead to an undesirable magnitude dependence of the variability measure $\chi^{2}$. Instead (and to overcome this), we finally used the following error model: because all reference stars of a target field are non-variable, the scatter (standard deviation $\sigma_{\text {rms }}$ ) in their light curves is a measure of the total photometric error at the corresponding magnitude. We plotted $\sigma_{\text {rms }}$ versus $\sigma_{\text {IRAF }}$ and fitted a first order polynomial to this graph. Applying this fit, i.e. $\sigma_{\text {tot }}=a+b \cdot \sigma_{\mathrm{IRAF}}$ where $a$ and $b$ are the fit's free parameters, we arrive at a reliable error description. Typical values of $a$ and $b$ are -0.01 to 0.01 and 0.5 to 1.5 , respectively. The fitting procedure was done for each field and channel separately. The reference star selection is an iterative process, which means that first the $\chi^{2}$ measure (see Sect. 4.1) is determined for all candidates. Then the most variable one is excluded from the set and the process is repeated until no variable star remains. Our error model partly accounts for varying data quality on different nights, e.g. due to bad seeing conditions, since this manifests itself as an increased standard deviation in the reference stars.

We further found that other informal errors contribute far less than $0.5 \%$. This includes imperfect overscan subtraction and two rather unusual sources: weak charge trailing on the CCD for brighter stars (and which appears only in the G-channel) and what we call glitches. These are quite similar in size and intensity to cosmics but are caused by the read-out electronics. They are quite numerous in the $I$-channel (some hundred per image) but only rare in $R$ and $G$.

\section{Time series analysis}

\section{1. $\chi^{2}$ test}

To test whether or not deviations in a target's relative light curve are consistent with the photometric errors (the null hypothesis) we evaluated the $\chi^{2}$ measure

$$
\chi^{2}=\sum_{i}^{N}\left(\frac{m_{\mathrm{rel}}(i)}{\delta m_{\mathrm{rel}}(i)}\right)^{2}
$$

where $m_{\text {rel }}(i)$ is the relative magnitude in the $i$ th (of $N$ ) frame and $\delta m_{\text {rel }}(i)$ the error therein. The larger the $\chi^{2}$ value, the larger the probability that the null hypothesis is wrong and that the object is variable. We claim an object to be variable if the probability for the null hypothesis $p$ is smaller than 0.01 . We used this test first to assemble the set of reference stars and then to test for possible variability within the targets.

We often encountered the case that significant general or periodic variability is only present in one or two channels. This can either be due to different sensitivity limits or to different variability amplitudes in the channels (or to a combination of both). As we will discuss in chapter 5, surface features can indeed lead to such a behaviour.

\subsection{Periodic variability}

Periodic variability can be caused by co-rotating surface features which are stable on time scales of an object's rotation period. To check for periodic behaviour, the following scheme was applied to each target: the CLEAN periodogram was calculated and searched for significant peaks; in case there is such a peak, the target's light curve was phased to the corresponding period and checked if it confirms the period in question. The Lomb-Scargle periodogram was also evaluated for all targets but the influence of the spectral window function is very strong for periods longer than approximately $10 \mathrm{~h}$. Because of this the Scargle power spectra are not used for the final results. Nevertheless, the shorter periods of 2M1707+64 and LHS370 are confirmed by this method.

The CLEAN algorithm tries to remove the influence of the discrete and finite sampling of observational data on power spectra. For more information on this, see BJM01 and Roberts (1987). To judge whether a peak in the power spectrum is statistically significant, we performed Monte-Carlo simulations to determine the false alarm probability (FAP) power levels for each time sampling. The FAP denotes the probability that a peak in the power spectrum is caused by noise. We chose to use the same methods as already discussed by Lamm et al. (2004). Artificial light curves of non-variable stars were created by simulating pure Gaussian noise as well as by shuffling the actual magnitudes of the target's light curve with respect to the real epochs. For both methods, the highest peak in the corresponding CLEAN periodogram was determined and the power level that was exceeded by 1000 out of the $10^{5}$ simulations was defined to be the $1 \%$ FAP power level (similarly one obtains the $10 \%$ and $0.1 \%$ levels). To be conservative we compare the corresponding two values obtained for both methods and use the larger one. We claim periodic variability if peaks above the $1 \%$ limit are present.

\subsubsection{Period uncertainty}

To investigate the uncertainty in the detected periods, we simulated a sinusoidal signal at random phase with respect to the time sampling and added Gaussian noise. We define the Amplitude Ratio (AR) to be the ratio between the root-measquare (rms) amplitude of the sinusoidal and the one of the noise. The absolute value of the difference between the period of the input signal and that of the highest peak in the CLEAN 


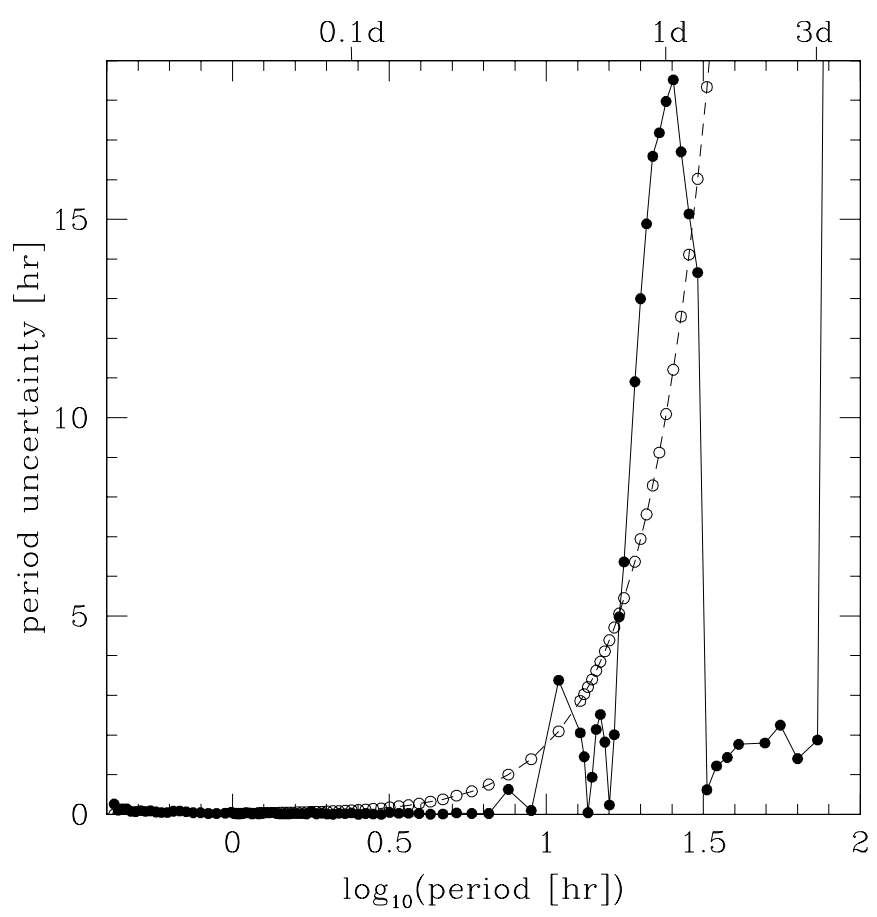

Fig. 2. Period uncertainty in hours plotted versus the logarithm of the period in hours; obtained for an amplitude ratio of 1.5 for the 2M1707+64 I time sampling with the method described in Sect. 4.2 (solid line and solid circles). The results of Eq. (1) are also included as open circles on a dashed line.

power spectrum was averaged over $10^{4}$ simulations at each input period. This result is a measure for the expected period uncertainty at the period of the sinusoid. An example for one time sampling is shown in Fig. 2. The input frequency is varied over the whole range of frequencies (actually we divided that range into 100 bins and performed $10^{4}$ simulations per bin). The shape of the curve is only weakly dependent on the AR for values above 1.5 and period uncertainties only slightly increase for ARs down to 1.0. Table 4 shows that, except for one case, all claimed periods occur at ARs of more than one. The simulations show that the period accuracy is very good for periods up to about $10 \mathrm{~h}$ and becomes much worse between 15 and $30 \mathrm{~h}$. This can be attributed to the gap in the data between the two consecutive observation nights. Although we expect the uncertainty to become lower for yet longer periods (since we observed the target on two nights), it is surprising how low it actually gets. A possible problem of these simulations is that they assume a sinusoidal signal, whereas periodic variability does not necessarily match this.

A similar procedure to the above has been used by Scholz \& Eislöffel $(2004 \mathrm{a}, \mathrm{b})$ who find a similar behaviour of the period uncertainty. Also shown in Fig. 2 (open circles) are the results of a theoretical period uncertainty estimate (for small errors):

$\Delta P \approx \frac{\Delta v P^{2}}{2}$.

Here $\Delta v$ is the width of the main peak of the window function $W(v)$, that can be approximated for not too uneven data sampling by $\Delta v \approx \frac{1}{T}$. Here, $T$ is the total time span covered by the observations. $P$ and $\Delta P$ are the period in question and its uncertainty, respectively. For long periods (larger errors) the approximation made to derive the above equation may no longer be valid. This would explain the huge difference relative to the results obtained with the simulations at longer periods. For more information on Eq. (1), see Roberts (1987).

Since the predicted uncertainties may differ by a factor of up to 3 between these two methods and since both methods do have drawbacks, it is difficult to decide which one to use. Thus the period uncertainties we state are always the results of the simulations but we also give the values obtained by Eq. (1) in parenthesis. Apart from just finding errors on the periods found with this survey, the period uncertainty is important when periods are reported in more than one channel of a target. If those periods are close to each other, it is likely that they actually correspond to a single periodicity, e.g. the rotation period, if their values do not differ by more than two or three times their uncertainty.

\subsubsection{Detection fraction}

To study the sensitivity of our period detection procedure, we introduce the detection fraction (DF). This quantity is equal to the fraction of detected periodic signals as a function of period; it is calculated using simulations of a sinusoidal and Gaussian noise (with the same definition of the AR as in the last section). The whole range of frequencies was divided in 100 bins and the simulations performed separately for each bin. The fraction of simulations with a fixed input frequency which lead to a peak in the power spectrum above the 1\% FAP power level is equal to the DF. We applied two different DFs, one where peaks anywhere in the periodogram were considered $\left(\mathrm{DF}_{\mathrm{all}}\right)$ and another where a significant peak was counted only if it was found inside the input bin $\left(\mathrm{DF}_{\mathrm{bin}}\right)$. Naturally, the detection fraction depends strongly on the AR. See Fig. 3 for the results on the same target as in Fig. 2. The resemblance to the plot of the period uncertainty is striking and naturally because both quantities depend on the same data sampling. Thus the same reasons can be given for the minimum in the DF at about $P=10-30 \mathrm{~h}$ and for the maximum in the period uncertainty at the same period range. The difference between the solid and the dashed line in Fig. 3 is the fraction of period detections at the wrong frequencies, i.e. outside the input bin. In agreement with the period uncertainty simulations, this difference is quite small at periods shorter than $10 \mathrm{~h}$ and thus if we detect a periodicity within this range it is very likely detected at the right period. If we again compare the ARs given in Table 4 with the Detection Fraction plots of the individual targets, we can summarise the results as follows: for most time samplings, an AR of 1.5 leads to a DF between $50 \%$ and $80 \%$; for ARs of 1.0 , the DF lies between $15 \%$ and $30 \%$ (in the case of $2 \mathrm{M} 1707+64$, see Fig. 3, the DFs are somewhat lower at each AR). Hence it is not unlikely that we missed periods for targets with ARs of less than 1.5, even in the range of up to ten hours. Furthermore, the $v \sin i$ distribution of $\mathrm{M}$ dwarfs (Mohanty \& Basri 2003; Delfosse et al. 1998) suggests that there are some objects, particularly at early $\mathrm{M}$ type, with expected periods of much more than $10 \mathrm{~h}$ (up to a few days). For these, the sensitivity of our detection procedure is questionable. 


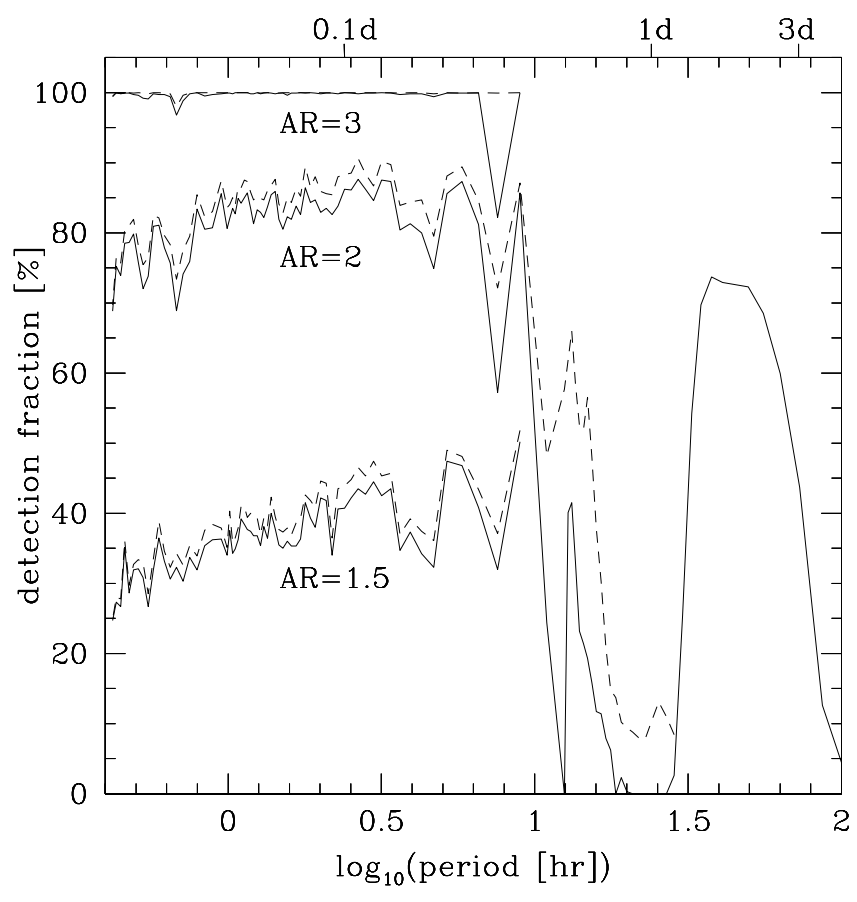

Fig. 3. The plot shows various detection fractions for the $2 M 1707+64$ time sampling over the logarithm of the period in hours. Solid lines represent detection fractions which require a peak to be found in a narrow range around the input period (i.e. inside the input bin), $\left(\mathrm{DF}_{\mathrm{bin}}\right)$, and dashed lines the ones that allow a peak to be at any period $\left(\mathrm{DF}_{\text {all }}\right)$. For periods of up to $\log _{10}$ (period) $=1.0$ three amplitude ratios are investigated: $3,2,1.5$ from top to bottom within the plot; for each ratio one solid and one dashed line. Because the general behaviour does not change, and to avoid crowding, for periods longer than ten hours only the curves corresponding to an amplitude ratio of 2 are shown. Here the solid and dashed lines merge at $\log _{10}($ period $)=1.47$.

Hence, to lower the probability of missing periods significantly, one would have to perform higher SNR observations and/or get a longer time base.

\subsection{Flares}

We will very briefly address the topic of flares in the observed field $\mathrm{M}$ dwarfs. For detailed information on the flares detected in this data set, see Rockenfeller et al. (2006).

Although well-known, the exact processes taking place during a flare event are not well-understood. Probably, magnetic energy is transfered to thermal energy and thus leads to a brightening of the effected area on or near the stellar surface. Flares on the Sun are often associated with eruptive promininences, sometimes ejecting solar material and charged particles into the solar system.

In principle, multiband monitoring in optical bands is ideal to detect flares because the amplitude of the brightness variations increases tremendously from the $I$ - to the $U V$-band. A flare is characterised by a fast rising signal followed by a slower decreasing one (of exponential shape). The duration of the event is positively correlated with its amplitude and ranges from a few minutes (or even shorter) to a few hours. This creates the problem that low-amplitude flares are too short to be seen in detail in this data set since the minimum time span between two data points is about $5 \mathrm{~min}$. However, one huge flare was detected in $2 \mathrm{M} 1707+64$, with an UV-amplitude of more than 6 mag and a recorded duration of about $1 \mathrm{~h}$. For this event we captured the brightness evolution over five data points. Three other events that are probably flares were found in $2 \mathrm{M} 1714+30,2 \mathrm{M} 1546+37$ and $2 \mathrm{M} 1344+77$ at lower amplitudes (between 1.4 and $1.7 \mathrm{mag}$ in UV). The total observation time of this survey is $218.0 \mathrm{~h}$, yielding a flare rate of $9.2 \times 10^{-4} \mathrm{~h}^{-1}, 1.83 \times 10^{-3} \mathrm{~h}^{-1}$ if we only count the two strongest events or all four, respectively.

\section{Results}

\subsection{General results}

The results of the $\chi^{2}$ test and the period search for all 19 targets are listed in Table 4. It shows the spectral type, the number of finally used reference stars $\left(N_{\mathrm{rs}}\right)$, the $\chi^{2}$ value of the light curve and the one corresponding to $p=0.01\left(\chi_{0}^{2}\right)$, variability flags, the probability of the null hypothesis $p_{\text {tot }}$, the variability amplitude, a possible period, the Amplitude Ratio and the finally claimed variability flag. Here, the variability amplitude is simply the root-mean-square (rms) of the relative light curve in case of variable objects and an estimation of an upper limit above which variability would have been detected for non-variable objects (using the method of BJM01, Sect. 5.1). The variability flags in Col. 6 state whether or not a target is variable according to the $\chi^{2}$ test on the individual observation nights (and in total in parentheses). In contrast to this, the flag in the last column indicates whether we finally claim the target to be variable, after consulting all means of investigation. We claim a target to be generally variable if the probability of the null hypothesis of the $\chi^{2}$ test is less than $1 \%$. This choice is quite arbitrary and using other limits would turn some detections into non-detections or vice versa. Likewise, periodic variability is claimed to be significant if the corresponding peak in the periodogram has a FAP of less than $1 \%$. In all cases where we found variability or remarkable features in a target's light curves, we also visually checked the reference stars' light curves, variability measures and power spectra to rule out exterior influences.

\subsection{Discussion of individual targets}

\section{LHS370 (M5)}

Due to bad seeing of up to $3^{\prime \prime}$ on night 8 in 2002, the star aperture radius had to be increased for LHS370 from 6 to 9 pixels to maximise the SNR. This object shows signs of variability in all three channels. In $I$ it is a strong detection $\left(p<10^{-6}\right)$ according to the $\chi^{2}$ test but a non-detection in $R$ and $G$. Nevertheless, the light curves (Fig. 4) of $R$ and $G$ show variations at similar amplitudes to that in $I$, but with larger errors. Although our data only cover about one cycle, the shape of the light curves suggests a periodic behaviour except for the $I$-band. This is confirmed since the power spectra do contain significant (above the $0.1 \%$ FAP power level) peaks in $R$ and $G$ at periods of $5.9 \pm 2.0 \mathrm{~h}$ 


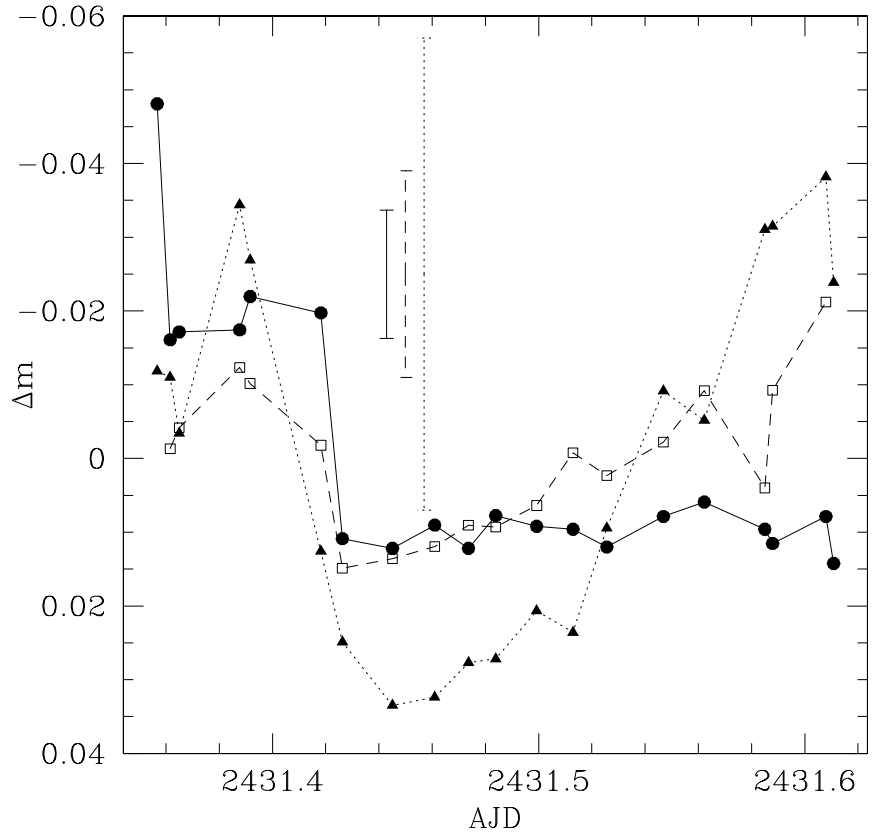

Fig. 4. Relative light curves of LHS370 at an aperture radius of nine pixels. The $I, R$ and $G$ band time series are shown with a solid, dashed and dotted line, respectively. Typical error bars are plotted for each channel.

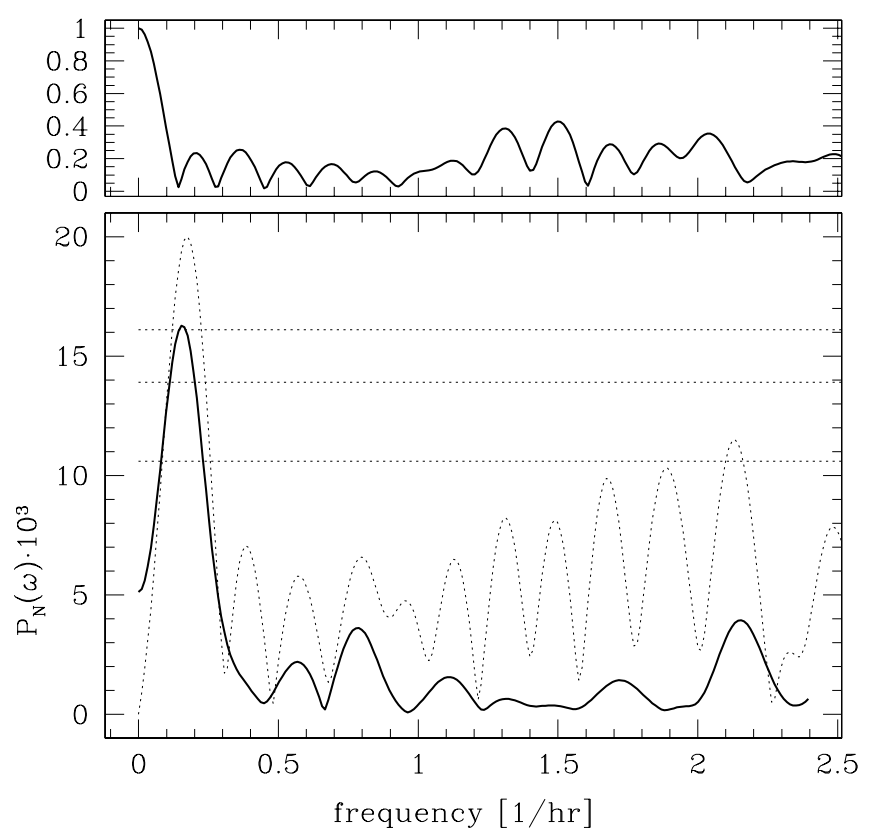

Fig. 5. CLEAN periodogram of the $G$-band data of LHS370. The upper panel shows the spectral window function and the lower panel the dirty power spectrum as a dotted line as well as the cleaned power spectrum as a thick solid line. The $0.1 \%, 1 \%$ and $10 \%$ FAP power levels are plotted as dotted horizontal lines (from top to bottom).

(theoretical uncertainty $2.9 \mathrm{~h})$ and $6.5 \pm 2.0 \mathrm{~h}( \pm 3.5 \mathrm{~h})$, respectively, but none in $I$. See Fig. 5 for the CLEAN periodogram of the $G$-band data and its light curve phased to the just mentioned period in Fig. 6. Since the phased light curves in both $R$ and $G$ look reasonable, there is good evidence for periodic variability. Studying the results of the period uncertainty simulations

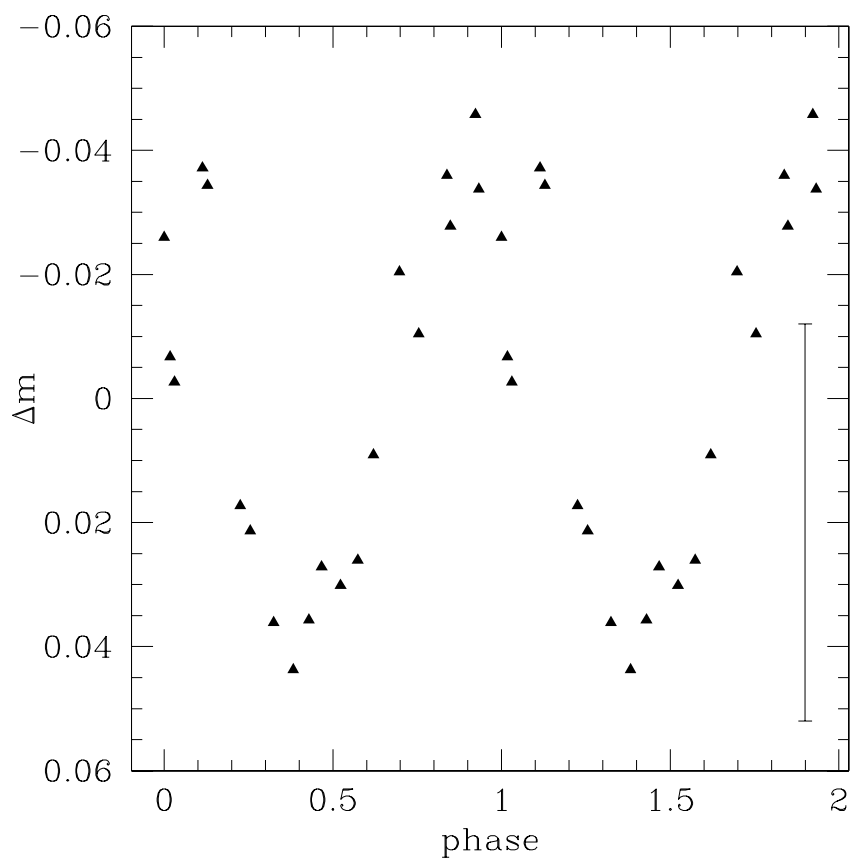

Fig. 6. $G$ band light curve of LHS 370 phased to a period of $6.5 \mathrm{~h}$. The typical error is also shown. Note that two cycles of the phased light curve are plotted.

(see above), we find that they are consistent with a single periodicity. Since the Amplitude Ratio (AR) of the I-band data is 2.4 we can confidently infer that there is no similar period in the $I$-band compared to those in $R$ and $G$. Because of the higher AR in $R$ (compared to $G$ ), we claim the $R$-band period at $5.9 \pm 2.0 \mathrm{~h}$ to be the object's rotation period. This is consistent with $v \sin i$ measurements of various M dwarfs by Mohanty \& Basri (2003) and Delfosse et al. (1998). To investigate possible sources for this kind of variability, we compared the observations with a grid of synthetic atmosphere spectra. For details on this procedure see the section on $2 \mathrm{M} 1707+64$ below. From such an analysis we could not match the observed variability amplitudes simultaneously in the three channels with any reasonable atmospheric model spectra featuring either magnetically induced star spots or dust clouds. Alternative models for dust clouds or spots may be required. We define the coverage factor $e$ as the fraction of the visible hemisphere that is covered by surface features. By simply comparing the predicted amplitudes with the observed, we can however place an upper limit of $e=0.05$ on the coverage factor of spots on the surface of LHS370. Otherwise we would have been able to clearly detect their signature in the $I$-band. Higher SNR data would yield a higher photometric precision and thus allow us to better constrain the source of variability in LHS370.

\section{M1707+64 (M9)}

This target shows various kinds of variability. First, it is a strong detection in terms of the $\chi^{2}$ test in $I$ and $R$ on both observation nights $\left(p<10^{-6}\right.$ and $p=0.001$, respectively) and only on night two in $G$. Besides this general variability indication, periods with FAPs better than $0.1 \%$ at $3.65 \mathrm{~h}, 3.7 \mathrm{~h}$ and 

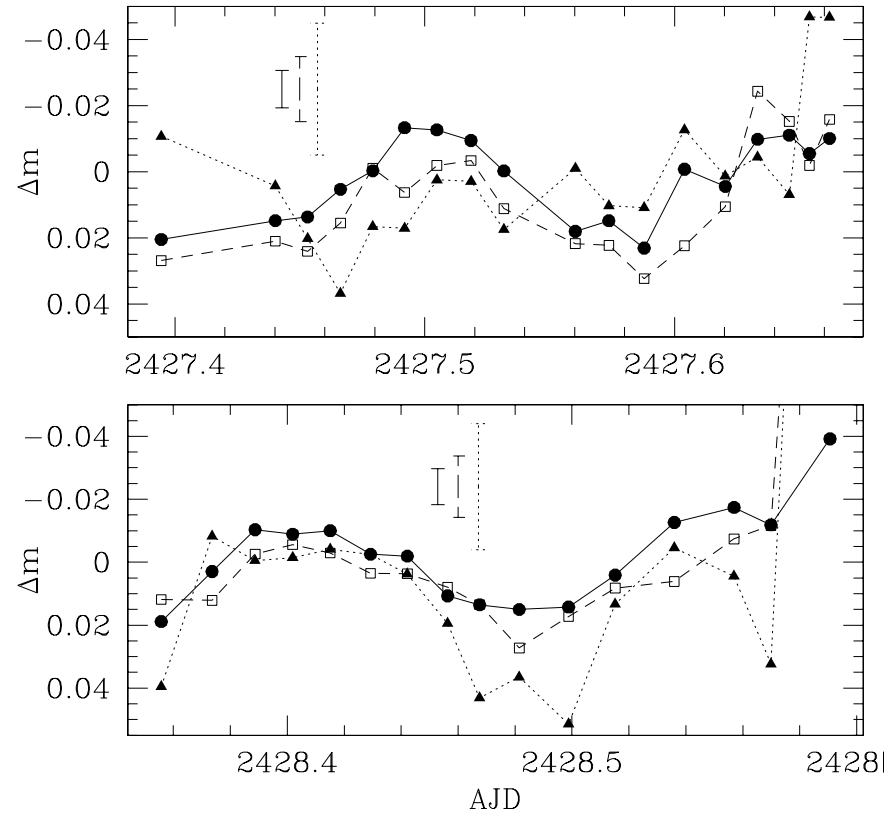

Fig. 7. The relative light curves of $2 \mathrm{M} 1707+64$ in $I$ (solid circles on a solid line), $R$ (open squares on a dashed line) and $G$ (solid triangles on a dotted line). Each observation night is shown in an individual panel with typical error bars for each channel.

$3.3 \mathrm{~h}$ are present in $I, R$ and $G$ respectively. The uncertainty in those periods is estimated to be about $0.1 \mathrm{~h}$ (theoretical estimate $0.6 \mathrm{~h}$ ) and thus all three values correspond to the same periodicity. As with LHS370, this period is consistent with the $v \sin i$ values of late $\mathrm{M}$ type objects, so we claim this to be the rotation period. Figure 7 shows that these periods can be seen visually as nearly sinusoidal modulations in the light curves. Figure 8 shows the CLEAN power spectrum in I and Fig. 9 the $I$ band light curve phased to the mentioned period. A huge flare was detected at the end of the second night which confirms that magnetic activity is significant in this late type object. For more information on this and other flares found within this data set, see Rockenfeller et al. (2006).

Since the variations in the light curves of the different channels are strongly correlated, it is obvious to assume that some surface feature co-rotating with the target is the reason for the measured period. To put stronger constraints on the source of variability in $2 \mathrm{M} 1707+64$, we used the synthetic atmospheric models of Allard et al. (2001). As shown in Bailer-Jones (2002), it is straight forward to derive the difference spectrum caused by co-rotating or forming / dissolving star spots or dust clouds. By integrating the predicted spectral variations over our photometric bands, we arrive at variability amplitudes for a specific model (refer to the just mentioned paper for a description of the two cases used, dusty and cond, as well as example spectra plots). Since, for a specific model, the predicted amplitudes depend linearly on the coverage factor $e$ of the surface features (for values of up to $e=0.3$ ), the ratio of two amplitudes in different wavebands will be independent of $e$. This makes these ratios suitable for assessing whether or not an individual model fits our data. Based on this, we built a grid of results for various trial atmospheric models.

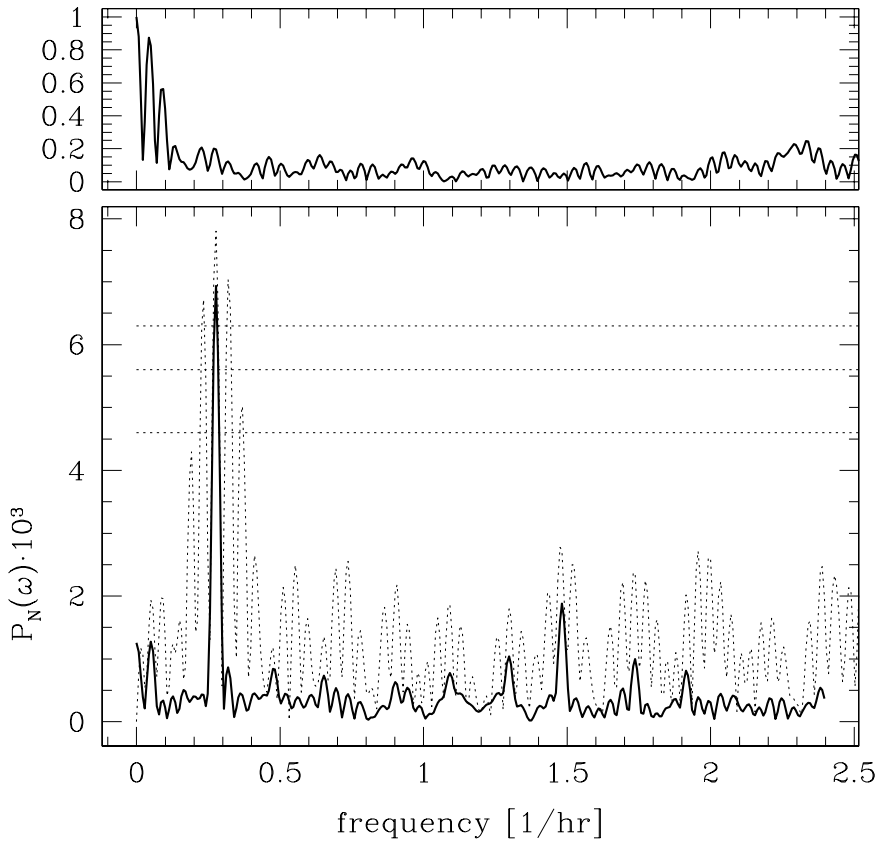

Fig. 8. CLEAN periodogram of the $I$ band light curve of 2 M1707+64. The upper panels shows the spectral window function and the lower panel the dirty power spectrum as a dotted line as well as the cleaned power spectrum as a thick solid line. The $0.1 \%, 1 \%$ and $10 \%$ FAP power levels are plotted as dotted horizontal lines (from top to bottom). Cf. Fig. 5. The above periodogram has higher resolution due to the longer time base.

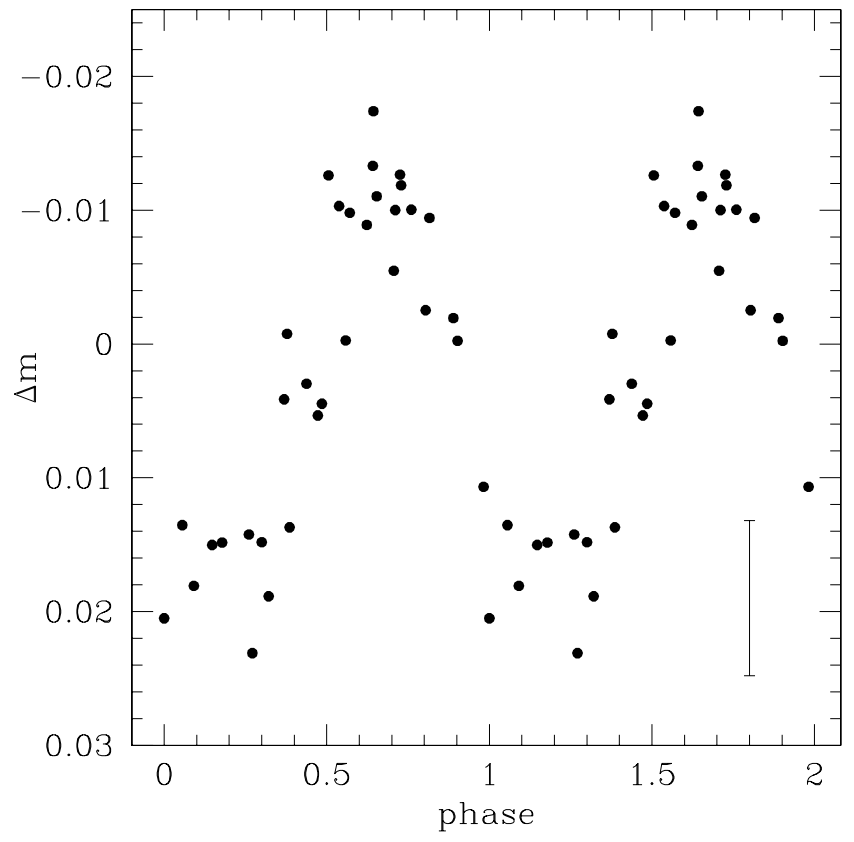

Fig. 9. Relative light curve of $2 \mathrm{M} 1707+64$ in I phased to a period of $3.65 \mathrm{~h}$. The plot shows the combined data of both nights. The typical error is also plotted. Note that two cycles of the phased light curve are plotted.

The best-fitting model (dusty atmosphere with $T_{\text {eff }}=2300 \mathrm{~K}$, $\log g=5.0$ and a $100 \mathrm{~K}$ cooler spot) along with the observational amplitudes are shown in Table 2, whereas Table 3 shows some other results from the computed model grid. 
Table 2. Comparison of observed and simulated variability amplitudes. The upper panel shows the observational standard (std), i.e. rms, and peak-to-peak (ptp) amplitudes and the ratios formed hereof. Ratio errors are estimated from the errors in the relative magnitudes. Theoretical amplitudes and ratios are presented in the lower part. The four cases are cool spot or dusty cloud on a cond (clear) atmospheres, and cool spot or a hole (cond "cloud") on a dusty atmosphere. The underlying atmosphere has an effective temperature of $2300 \mathrm{~K}$ and surface gravity of $\log g=5.0$; the cool spot is $100 \mathrm{~K}$ cooler and all feature types (spot or cloud) have a projected surface coverage factor of 0.1 . These are the parameters of the best-fitting model. Values in parentheses indicate the amplitude ratios as explained in the last column. Note that the amplitude ratios are independent of the coverage factor for small $(<0.3)$ coverage factors.

\begin{tabular}{lllllll}
\hline \hline & \multicolumn{3}{c}{ Observational quantities } & & \\
Band / colour & std ampl. [mag] & ptp ampl. [mag] & ratio & std ratio & ptp ratio & ratio error \\
\hline$G$ & 0.022 & 0.055 & $G: R$ & 1.57 & $\mathbf{1 . 4 9}$ & \pm 0.25 \\
$R$ & 0.014 & 0.037 & $G: I$ & 1.83 & $\mathbf{1 . 6 7}$ & \pm 0.25 \\
$I$ & 0.012 & 0.033 & $R: I$ & 1.17 & $\mathbf{1 . 1 2}$ & \pm 0.30 \\
\hline
\end{tabular}

\begin{tabular}{lllllc}
\hline & \multicolumn{4}{c}{ Theoretical quantities } & \\
Band / colour & cool spot & dusty cloud & cool spot & cond cloud & legend \\
ampl. (ratio) \\
\hline$G$ & $0.039(1.56)$ & $0.064(1.42)$ & $0.072(\mathbf{1 . 4 8})$ & $-0.135(1.85)$ & $G(G: R)$ \\
$R$ & $0.025(1.56)$ & $0.045(3.05)$ & $0.049(\mathbf{1 . 7 0})$ & $-0.073(5.40)$ & $R(G: I)$ \\
$I$ & $0.025(1.00)$ & $0.021(2.14)$ & $0.042(\mathbf{1 . 1 5})$ & $-0.025(2.92)$ & $I(R: I)$ \\
\hline
\end{tabular}

Table 3. Supplementary data (to Table 2) that shows the amplitude ratios as calculated with model parameters given in the first two columns (the surface gravity is fixed at $\log g=5.0$ since it does not have a significant influence on the results; feature sizes are independent of the coverage factor up to $e \approx 0.3$ ). cond spot stands for a cool cond spot on a cond background atmosphere and dusty cloud similarly denotes a dusty cloud on a cond atmosphere.

\begin{tabular}{|c|c|c|c|c|c|c|c|c|c|}
\hline \multicolumn{10}{|c|}{ Grid of theoretical quantities - amplitude ratios } \\
\hline \multicolumn{2}{|c|}{ Model parameters } & \multicolumn{4}{|c|}{ Cond atmosphere } & \multicolumn{4}{|c|}{ Dusty atmosphere } \\
\hline$T_{\text {eff }}[\mathrm{K}]$ & $(\Delta T)_{\text {spot }}[\mathrm{K}]$ & & $G: R$ & $G: I$ & $R: I$ & & $G: R$ & $G: I$ & $R: I$ \\
\hline \multirow[t]{2}{*}{2300} & 100 & cond spot & 1.56 & 1.56 & 1.00 & dusty spot & 1.48 & 1.70 & 1.15 \\
\hline & & dusty cloud & 1.42 & 3.05 & 2.14 & cond cloud & 1.85 & 5.40 & 2.92 \\
\hline 2300 & 200 & cond spot & 1.62 & 1.38 & 0.85 & dusty spot & 1.24 & 1.33 & 1.07 \\
\hline 2300 & 300 & cond spot & 1.60 & 1.24 & 0.77 & dusty spot & 1.14 & 1.16 & 1.02 \\
\hline \multirow[t]{2}{*}{2500} & 100 & cond spot & 1.22 & 1.92 & 1.57 & dusty spot & 1.27 & 1.96 & 1.54 \\
\hline & & dusty cloud & 1.44 & 6.82 & 4.73 & cond cloud & 1.59 & 8.70 & 5.46 \\
\hline 2500 & 200 & cond spot & 1.22 & 1.70 & 1.39 & dusty spot & 1.21 & 1.66 & 1.38 \\
\hline 2500 & 300 & cond spot & 1.22 & 1.49 & 1.22 & dusty spot & 1.15 & 1.38 & 1.20 \\
\hline \multirow[t]{2}{*}{2100} & 100 & cond spot & 2.25 & 1.16 & 0.51 & dusty spot & 1.36 & 1.23 & 0.91 \\
\hline & & dusty cloud & 1.21 & 1.60 & 1.33 & cond cloud & 2.57 & 5.50 & 2.14 \\
\hline
\end{tabular}

The theoretical predictions were made for cooler spots on either type of background atmosphere (cond and dusty) as well as for clouds of the opposite type and the same temperature as the background atmosphere, i.e. a cond cloud on a dusty atmosphere (clear hole in a dusty sky) and the other way around (dusty cloud on a clear sky). This setup excludes fast convection as a source for the clouds since they would then be hotter than the surrounding regions. Because of their small size (compared to the noise in individual measurements), the determination of the observational amplitudes is non-trivial. We decided to form both the peak-to-peak amplitudes (ptp) after an exclusion of obvious outliers and the root-mean-square of the light curve, here called standard amplitude (std). In the case of $2 \mathrm{M} 1707+64$, the amplitude ratios formed with std and ptp amplitudes are similar because of the nearly sinusoidal shape of the light curves. For a perfectly sinusoidal signal the std amplitude is $\sqrt{\pi} / 2$ times the ptp amplitude.
Studying both tables for $T_{\text {eff }}=2300 \mathrm{~K}$ and $T_{\text {eff }}=2500 \mathrm{~K}$, we can clearly exclude clouds as the source of variability because the predicted signatures are too far from the measured ones. The case of a dusty cloud on a $T_{\text {eff }}=2100 \mathrm{~K}$ cond atmosphere also is an acceptable fit to the data. But first, the effective temperature is somewhat lower than the one derived by the spectral type (Gorlova et al. 2003, although the uncertainty in $T_{\text {eff }}$ is about $200 \mathrm{~K}$ ) and second, and more convincingly, cond atmospheres are expected to occur not earlier than T-type objects.

Besides the best-fitting one, models with spots on (particularly dusty) atmospheres of $2200 \leq T_{\text {eff }} \leq 2500$ fit the observed amplitude ratios within the estimated errors. Hence we conclude that co-rotating magnetically induced spots, most likely on a dusty atmosphere of 2200-2500 K, cause the observed variability in $2 \mathrm{M} 1707+64$. We infer from the observed amplitudes that the coverage factor of these spots cannot be larger 
Table 4. Summary of important results: $N_{r s}$ is the number of reference stars, $\chi^{2} / \chi_{0}^{2}$ the actual and critical $\chi^{2}$ values (i.e. for $p=0.01$ ), var. flags indicates whether a target is variable on the individual nights and in total (the latter in parenthesis) according to the $\chi^{2}$ test, $p_{\text {tot }}$ is the probability of the null hypothesis, ampl. the variability amplitude (root-mean-square) or an upper limit hereto (both in magnitudes), period is self-explanatory (measured in hours with uncertainties obtained by the simulations, see Sect. 4.2.1, although note that theoretical values are larger); AR (Amplitude Ratio) is the ratio of the rms amplitude of the light curve to the noise within the target; var. states whether or not a channel was finally considered variable. The + and - flags indicate variable and non-variable objects, respectively, whereas o means that it is only a marginal detection (either in terms of the $\chi^{2}$ test or of a consideration of all means of investigation).

\begin{tabular}{|c|c|c|c|c|c|c|c|c|c|c|}
\hline Target & SpT & Band & $N_{\mathrm{rs}}$ & $\chi^{2} / \chi_{0}^{2}$ & var. flags & $p_{\text {tot }}$ & Ampl. & Period & AR & var. \\
\hline \multirow[t]{3}{*}{$2 \mathrm{M} 1311+80$} & M8 & $I$ & 17 & $38.3 / 54$ &,- o (-) & 0.21 & $0.012^{a}$ & - & 1.2 & - \\
\hline & & $R$ & 12 & $20.1 / 26$ & $-(-)$ & 0.065 & $0.020^{a}$ & - & 1.4 & - \\
\hline & & $G$ & 13 & $58.5 / 54$ &,$--(+)$ & $2.9 \times 10^{-4}$ & $0.088^{b}$ & - & 1.4 & - \\
\hline \multirow[t]{3}{*}{ 2M1336+47 } & M7 & $I$ & 9 & $72 / 95$ &,$--(-)$ & 0.71 & $0.0090^{a}$ & - & 1.3 & - \\
\hline & & $R$ & 10 & $66.9 / 95$ &,$--(-)$ & 0.41 & $0.022^{a}$ & - & 1.0 & - \\
\hline & & $G$ & 9 & $62.2 / 96$ &,$--(-)$ & 0.61 & $0.063^{a}$ & - & 0.8 & - \\
\hline \multirow[t]{3}{*}{$2 \mathrm{M} 1344+77$} & M7 & $I$ & 33 & $63.5 / 64$ &,$-+(\mathrm{o})$ & 0.011 & $0.0057^{a}$ & - & 2.0 & o \\
\hline & & $R$ & 18 & $51.8 / 58$ &,$--(-)$ & 0.034 & $0.016^{a}$ & - & 1.1 & - \\
\hline & & $G$ & 19 & $28.7 / 64$ &,$--(-)$ & 0.91 & $0.048^{a}$ & $12.5 \pm 3.0 ?$ & 0.6 & - \\
\hline \multirow[t]{3}{*}{ LHS370 } & M5 & $I$ & 14 & $57.7 / 37$ & + & $<10^{-6}$ & $0.015^{b}$ & - & 2.4 & + \\
\hline & & $R$ & 13 & $19.7 / 34$ & - & 0.29 & $0.020^{a}$ & $5.9 \pm 2.0$ & 1.2 & + \\
\hline & & $G$ & 14 & $16.1 / 37$ & - & 0.65 & $0.045^{a}$ & $6.5 \pm 2.0$ & 1.1 & + \\
\hline \multirow[t]{3}{*}{ LHS2930 } & M6.5 & $I$ & 10 & $80 / 85$ &,$+-(-)$ & 0.024 & $0.026^{a}$ & $13.2 \pm 1.9 ?$ & 2.4 & o \\
\hline & & $R$ & 16 & $55 / 85$ &,$--(-)$ & 0.53 & $0.0088^{a}$ & - & 2.1 & - \\
\hline & & $G$ & 10 & $56 / 85$ &,$--(-)$ & 0.52 & $0.063^{a}$ & - & 1.1 & - \\
\hline \multirow[t]{3}{*}{ CTI1539+28 } & M2 & $I$ & 29 & $74.9 / 75$ &,- o (o) & 0.01 & $0.0074^{a}$ & - & 2.8 & o \\
\hline & & $R$ & 21 & $40.9 / 75$ &,$--(-)$ & 0.79 & $0.0063^{a}$ & - & 0.8 & - \\
\hline & & $G$ & 15 & $67.0 / 74$ &,$--(-)$ & 0.036 & $0.014^{a}$ & - & 1.4 & - \\
\hline \multirow[t]{3}{*}{$2 \mathrm{M} 1546+37$} & M7.5 & $I$ & 25 & $58.7 / 75$ &,,$---(-)$ & 0.84 & $0.0063^{a}$ & - & 1.2 & - \\
\hline & & $R$ & 23 & $105 / 75$ &,,$++-(+)$ & $5 \times 10^{-6}$ & $0.012^{b}$ & - & 2.7 & + \\
\hline & & $G$ & 18 & $75 / 75$ &,,$---(\mathrm{o})$ & 0.01 & $0.025^{a}$ & - & 1.8 & - \\
\hline \multirow[t]{3}{*}{ LHS3189 } & M5.5 & $I$ & 35 & $59.3 / 74$ & $\mathrm{o},-(-)$ & 0.13 & $0.0056^{a}$ & - & 1.1 & - \\
\hline & & $R$ & 27 & $35.6 / 74$ &,$--(-)$ & 0.91 & $0.0097^{a}$ & - & 0.7 & - \\
\hline & & $G$ & 18 & $22.2 / 74$ &,$--(-)$ & 0.9995 & $0.032^{a}$ & - & 0.6 & - \\
\hline \multirow[t]{3}{*}{$2 \mathrm{M} 1627+81$} & M9 & $I$ & 36 & $56.8 / 78$ &,,$---(-)$ & 0.37 & $0.011^{a}$ & - & 1.0 & - \\
\hline & & $R$ & 31 & $74.0 / 78$ &,,$--+(-)$ & 0.019 & $0.024^{a}$ & - & 1.4 & - \\
\hline & & $G$ & 37 & $41.3 / 78$ &,,$---(-)$ & 0.83 & $0.051^{a}$ & - & 0.8 & - \\
\hline \multirow[t]{3}{*}{ CTI1629+28 } & M4 & $I$ & 28 & $44.1 / 74$ &,$--(-)$ & 0.64 & $0.012^{a}$ & - & 1.0 & - \\
\hline & & $R$ & 19 & $206 / 74$ &,$++(+)$ & $<10^{-6}$ & $0.014^{b}$ & - & 1.9 & + \\
\hline & & $G$ & 20 & $86.8 / 73$ &,$+-(+)$ & $4 \times 10^{-4}$ & $0.022^{b}$ & - & 1.5 & - \\
\hline \multirow[t]{3}{*}{$2 \mathrm{M} 1707+64$} & M9 & $I$ & 37 & $143 / 54$ &,$++(+)$ & $<10^{-6}$ & $0.012^{b}$ & $3.65 \pm 0.1$ & 2.5 & + \\
\hline & & $R$ & 32 & $62 / 54$ &,$++(+)$ & 0.001 & $0.014^{b}$ & $3.7 \pm 0.1$ & 1.7 & + \\
\hline & & $G$ & 25 & $50 / 54$ &,$-+(-)$ & 0.022 & $0.029^{a}$ & $3.3 \pm 0.1$ & 1.8 & + \\
\hline \multirow[t]{3}{*}{ CTI1709+27 } & M5.5 & $I$ & 43 & $120 / 80$ &,,$--+(+)$ & $<10^{-6}$ & $0.0062^{b}$ & - & 2.1 & + \\
\hline & & $R$ & 26 & $277 / 80$ &,,$-++(+)$ & $<10^{-6}$ & $0.011^{b}$ & - & 2.2 & + \\
\hline & & $G$ & 29 & $140 / 78$ &,,$+++(+)$ & $<10^{-6}$ & $0.014^{b}$ & - & 1.6 & + \\
\hline \multirow[t]{3}{*}{$2 \mathrm{M} 1714+30$} & M6.5 & $I$ & 32 & $30.0 / 77$ &,$--(-)$ & 0.99 & $0.0080^{a}$ & - & 0.8 & - \\
\hline & & $R$ & 31 & $107 / 77$ &,$++(+)$ & $<10^{-6}$ & $0.012^{b}$ & $6.9 \pm 0.15 ?$ & 1.9 & + \\
\hline & & $G$ & 27 & $144 / 77$ &,$++(+)$ & $5 \times 10^{-6}$ & $0.034^{b}$ & - & 1.8 & - \\
\hline \multirow[t]{3}{*}{ LHS3307 } & M5 & $I$ & 49 & $67.5 / 84$ &,$--(-)$ & 0.13 & $0.052^{a}$ & - & $>3$ & - \\
\hline & & $R$ & 50 & $104 / 84$ &,$+-(+)$ & 0.0001 & $0.012^{b}$ & - & 1.2 & o \\
\hline & & $G$ & 47 & $57.7 / 84$ &,$--(-)$ & 0.41 & $0.031^{a}$ & - & 0.8 & - \\
\hline \multirow[t]{3}{*}{$2 \mathrm{M} 1750+44$} & M7.5 & $I$ & 31 & $84.3 / 92$ &,$-+(-)$ & 0.038 & $0.0075^{a}$ & - & 1.1 & - \\
\hline & & $R$ & 36 & $62.4 / 88$ &,$--(-)$ & 0.36 & $0.018^{a}$ & - & 1.3 & - \\
\hline & & $G$ & 33 & $69.7 / 80$ & $o,-(-)$ & 0.061 & $0.036^{a}$ & - & 1.2 & - \\
\hline \multirow[t]{3}{*}{ LHS3339 } & M6 & $I$ & 32 & $116 / 75$ &,$--(+)$ & $<10^{-6}$ & $0.0061^{b}$ & - & 1.7 & + \\
\hline & & $R$ & 31 & $67.6 / 74$ &,$--(-)$ & 0.033 & $0.0083^{a}$ & - & 1.5 & - \\
\hline & & $G$ & 20 & $119 / 75$ &,$--(+)$ & $<10^{-6}$ & $0.021^{b}$ & - & 1.3 & + \\
\hline
\end{tabular}


Table 4. continued.

\begin{tabular}{lllllllllll}
\hline \hline Target & SpT & Band & $N_{\mathrm{rs}}$ & $\chi^{2} / \chi_{0}^{2}$ & var. flags & $p_{\text {tot }}$ & Ampl. & Period & AR & var. \\
\hline 2M1757+70 & M7.5 & $I$ & 50 & $48.6 / 60$ &,$--(-)$ & 0.097 & $0.0061^{a}$ & - & 4.7 & - \\
& & $R$ & 39 & $54.8 / 60$ &,$--(-)$ & 0.03 & $0.0090^{a}$ & - & 1.5 & - \\
CTI1801+28 & \multirow{2}{*}{ M2.5 } & $I$ & 31 & $49.0 / 84$ &,$--(-)$ & 0.73 & $0.0061^{a}$ & - & 1.1 & - \\
& & $R$ & 31 & $73.7 / 81$ &,$--(-)$ & 0.039 & $0.0055^{a}$ & - & 1.2 & - \\
& & $G$ & 27 & $63.2 / 81$ &,$--(-)$ & 0.18 & $0.015^{a}$ & - & 1.1 & - \\
LHS3376 & \multirow{2}{*}{ M4 } & $I$ & 44 & $135 / 50$ & + & $<10^{-6}$ & $0.052^{b}$ & - & $>3$ & - \\
& & $R$ & 25 & $27 / 50$ & - & 0.43 & $0.029^{a}$ & - & 1.3 & - \\
& & $G$ & 40 & $45 / 50$ & - & 0.029 & $0.050^{a}$ & - & 1.9 & - \\
\hline
\end{tabular}

Amplitude column: ${ }^{a}$ upper-limit-rms; ${ }^{b}$ light curve rms.
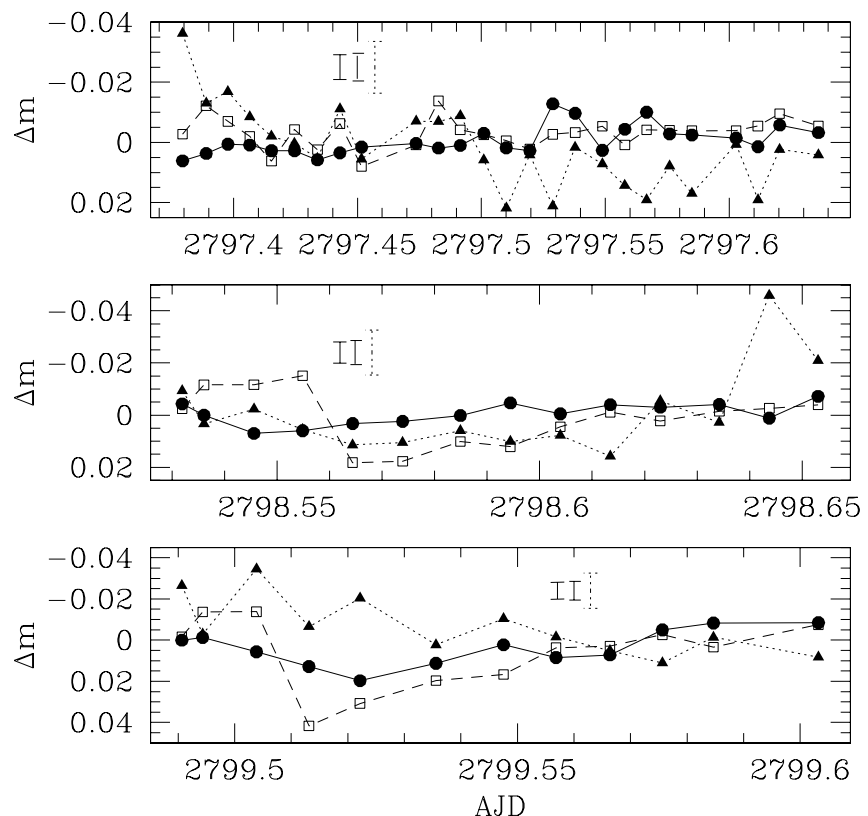

Fig. 10. Relative light curves of CTI1709+27 in the $I$-band (solid line), $R$-band (dashed line) and $G$-band (dotted line). Each observation night is shown in an individual panel with typical error bars for each channel.

than about $e=0.075$ for a dusty atmosphere (and about 0.15 for a cond one). Note that a coverage factor of e.g. $e=0.1$ does not necessarily refer to a single spot covering ten percent of the visible hemisphere. One can imagine other spot configurations leading to the same spectral signature as that of a single spot. For example, a symmetrical distribution of many small spots (with the same $e$ ) will not result in any detectable photometric signal. Thus the value of $e=0.075$ found for $2 \mathrm{M} 1707+64$ could also mean that a larger fraction of the star's hemisphere is covered by symmetrically distributed spots and that there is a non-symmetric spot coverage of about $7.5 \%$.

\section{CTI1709+27 (M5.5)}

We present the relative light curves of this target in Fig. 10 as an example for a typical object showing general variability above a probability of $99 \%$ (here even at $p<10^{-6}$, see Table 4 ) according to the $\chi^{2}$ test. No remarkable features or periodicities are present.

The choice of the probability at which to claim a target to be variable in terms of the $\chi^{2}$ test is quite arbitrary. The use of $p=0.05$ instead of $p=0.01$ would have led to 7 more targets to be claimed variable (at least in one channel each)! Thus one should first use a fairly low value for $p$ and second, it would be ideal to re-monitor those targets after some time to test for persistent variability. Of course, surface features could appear only temporarily and thus lead to a non-detection during the re-monitoring, even though they actually were present on the first observation run. Finally, this problem can be overcome by higher SNR data that permit even lower amplitude detections.

\section{Further variable objects}

Hints of periodic variability were also found in $2 \mathrm{M} 1344+77$ in $G(12.5 \pm 3.0 \mathrm{~h})$, in LHS2930 in $I(13.2 \pm 1.9 \mathrm{~h})$ and in $2 \mathrm{M} 1714+30$ in $R(6.9 \pm 0.15 \mathrm{~h})$ by the evaluation of the CLEAN power spectra, although the the visual appearance of the phased light curves do not support the presence of such periods. On the other hand, simulations of periodic modulations show that we should not always expect this, especially with multiple spots: see Sect. 6.2 of BJM01. Hence Table 4 lists these periods with a question mark as these periods are somewhat tentative. Of these three targets only $2 \mathrm{M} 1714+30$ (in $R$ ) is a detection according to the $\chi^{2}$ test.

General variability according to the $\chi^{2}$ test was detected in other targets as well, but they do not show any remarkable features. These targets are: CTI1539+28 $(I), 2 \mathrm{M} 1546+37(R)$, CTI1629+28 ( $R$ and $G), \operatorname{LHS3307}(R), \operatorname{LHS3339}(I$ and $G)$, LHS3376 $(I)$. Variability in the $G$-band data of CTI1629+28 was rejected because the two targets which were also observed in the same nights show similar trends in their corresponding $G$-band light curves. Because of bad data quality in the case of LHS3376, the variations in $I$ also cannot be considered to be significant.

Significant correlations between different channels could only be found for $2 \mathrm{M} 1707+64$ and LHS370, where they are obvious. Correlations are another indicator of the reliability of a variability. Of course, the underlying physical process could 
produce polychromatic variations such that the signal is too small to be detected in one or more channels.

In total, we find non-periodic variability in 5 out of $19 \mathrm{M}$ dwarfs (or 4, if we consider the period of $2 \mathrm{M} 1714+30$ to be real). Periodic variability was convincingly found in 2 targets (or up to 5, if we choose to be less conservative). Although these results are surely affected by small number statistics, they suggest that about $2 / 7 \simeq 30 \%$ of variable field $\mathrm{M}$ dwarfs show periodic variability.

The fact that we did not detect any variability for 12 out of 19 targets means that either no detectable surface features are present on these objects or that their rotation periods are too long to be detected. The latter may be particularly relevant for the four earliest type objects in our sample with spectral types M2-M4, since rotation periods of up to a few days are possible. The large fraction of non-periodic variable targets ( 5 out of 7 in total) could be due to rapidly evolving surface features which would veil the rotation period (the "masking hypothesis" of BJM01).

It is interesting to note that in the case of LHS370, no periodic variability was found in the $I$-band even though we assign a rotation period to this target on account of the $R$ - and $G$-band data. Hence we would have counted LHS370 to be generally variable but non-periodic if it were only observed in the $I$-band. Since almost all recent monitoring programs on UCDs have been conducted only in the $I$-band, it is possible that the large number of non-periodic variable $\mathrm{L}$ dwarfs found would show periods at other wavelengths. Multichannel observations of $\mathrm{L}$ dwarfs are required to investigate this.

\section{Non-variable objects}

All other targets show no signs of variability or exceptional behaviour and are hence not mentioned individually. At the present sensitivity limit, we consider them non-variable. See Table 4 for upper limits on variability amplitudes and for ARs. The latter allows us to judge whether we could have missed existing periods because of this, see Sect. 4.2.2.

\subsection{Follow-up observations of $2 M 1707+64$}

Follow-up observations on $2 \mathrm{M} 1707+64$, also using the BUSCA instrument, were performed as Directors Discretionary Time observations on the 14th and 15th of June 2005. The data reduction and analysis were done in the same way as for the 2002 and 2003 data. The relative light curves of $I, R$ and $G$ are shown in Figs. 11 and 12. All three channels are detections according to the $\chi^{2}$ test. Although the $R$ - and $G$-band data seems to consist of large random-like variations, the $I$-band light curve strongly resembles that from the 2002 data. Moreover, the $I$-band power spectrum contains a peak above the $0.1 \%$ FAP power level at $3.61 \pm 0.2 \mathrm{~h}$ which confirms the one we detected in the earlier data. This period can also be found on night two of the G-data $(P=3.80 \pm 0.2 \mathrm{~h})$ at the same significance and on night one with $P=4.0 \pm 0.27 \mathrm{~h}$ at a FAP of $1 \%$. The constancy of the period suggests that this is indeed a rotation period. We perhaps cannot say the rotation

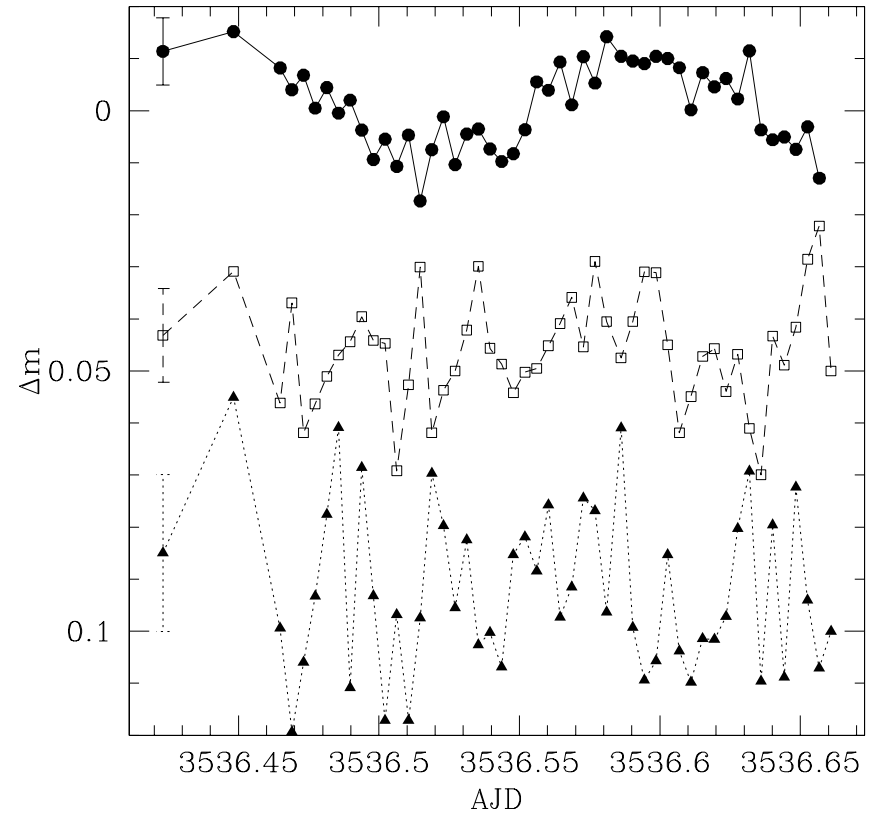

Fig. 11. The relative light curves of the first night of follow-up observations of $2 \mathrm{M} 1707+64$ with BUSCA in June 2005. $I$-band data is represented by solid lines, $R$-band data by dashed lines and $G$-band data by dotted lines. Error bars are shown only for one data point per channel.

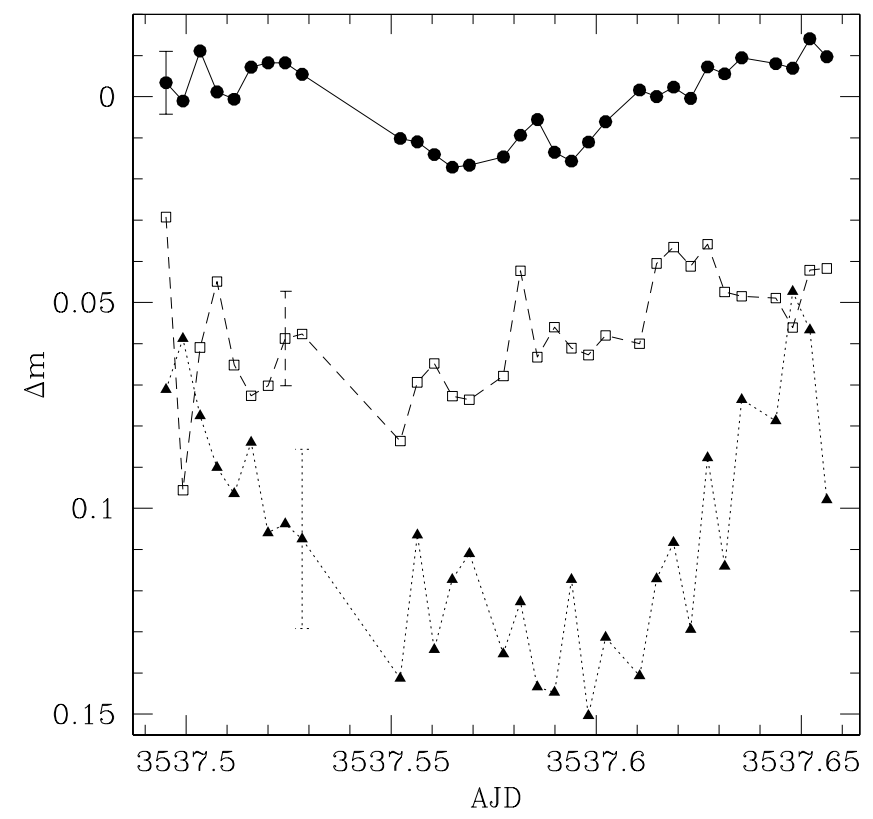

Fig. 12. The relative light curves of the second night of follow-up observations on $2 \mathrm{M} 1707+64$ with BUSCA in June 2005. $I$-band data is represented by solid lines, $R$-band data by dashed lines and $G$-band data by dotted lines. Error bars are shown only for one data point per channel.

period as the object may show differential rotation, although if so, the spots would have to be at similar latitudes (and/or the differential rotation would have to be small). Due to the lack of a detected period in $R$, we could not repeat the model spectra procedure to investigate the source of variability. 


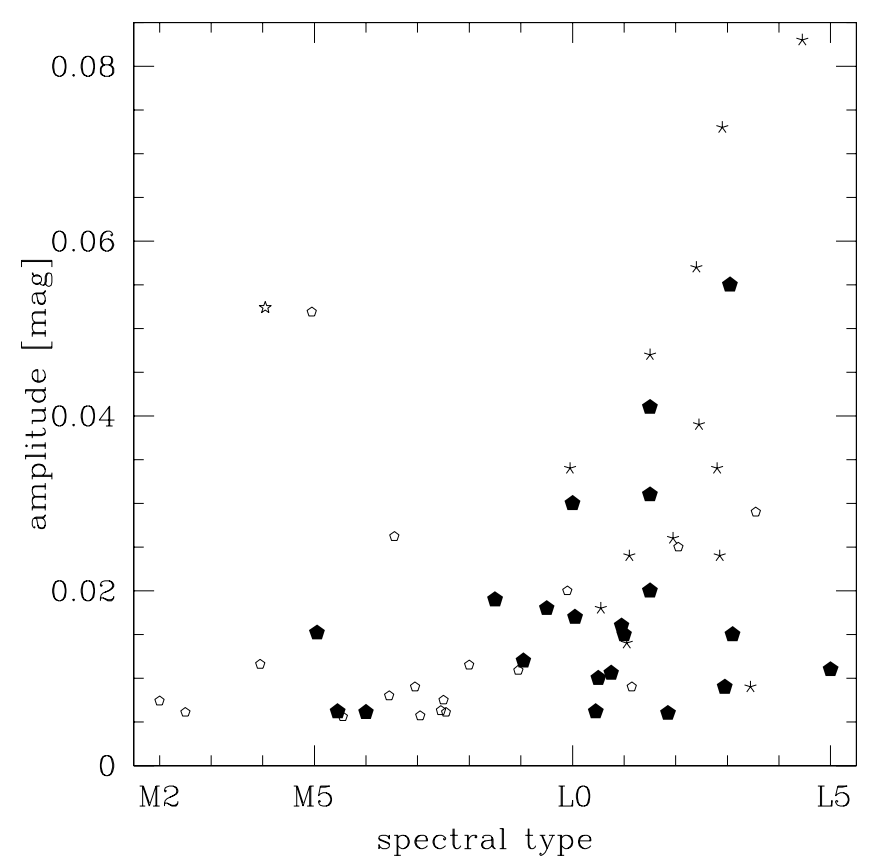

Fig. 13. Variability amplitudes (variable objects, solid markers) and upper detection limits (non-variable objects, open markers) compiled from all relevant works on field dwarfs in the $I$-band of the recent years, including: this paper, Bailer-Jones \& Mundt (2001), Clarke et al. (2002, 2003), Gelino et al. (2002), Koen (2005), Martín et al. (2001). The spectral types are slightly offset from the real ones in case there is more than one object of the same type. Amplitudes and upper limits are always rms values. Since Clarke et al. (2002) give peak-topeak amplitudes for their two variable targets, we converted those to rms values assuming a sinusoidal signal (that assumption is not entirely justified, but differences in amplitudes are less than fifteen percent). Skeletal markers indicate rms amplitudes of non-variable objects where no upper limits are available. Starred symbols stand for rms-amplitudes where the $\chi^{2}$ test gives a detection but variability is finally rejected.

Further independent observations on 2M1707+64 were carried out at the Maidanak Observatory, Uzbekistan, in the $I$-band in June 2005, as reported in Rockenfeller et al. (2006). These observations were made primarily to further investigate the flare activity of this target, but they also strongly support the rotation period, detected in the Maidanak data at $3.63 \pm 0.08 \mathrm{~h}$.

\subsection{Variability dependences}

One of the major goals of this survey was to study whether variability is more common in L dwarfs than in M dwarfs. To judge this, we compiled a list of recently observed (1999-2005) M and $\mathrm{L}$ field dwarfs from various publications (for sources, see the caption of Fig. 13). A direct comparison of data published by different groups is ambiguous since different detection limits were achieved or different significance levels were used. We therefore only use those data which we judge to be comparable to ours. Figure 13 shows the $I$ band variability amplitudes (or upper limits for non-variable objects, if available) versus the spectral type for these samples. Figure 14 shows variability amplitudes and upper limits of the $I-, R$ - and $G$-band data for all 19 objects of our data set. No definite trend of the amplitudes

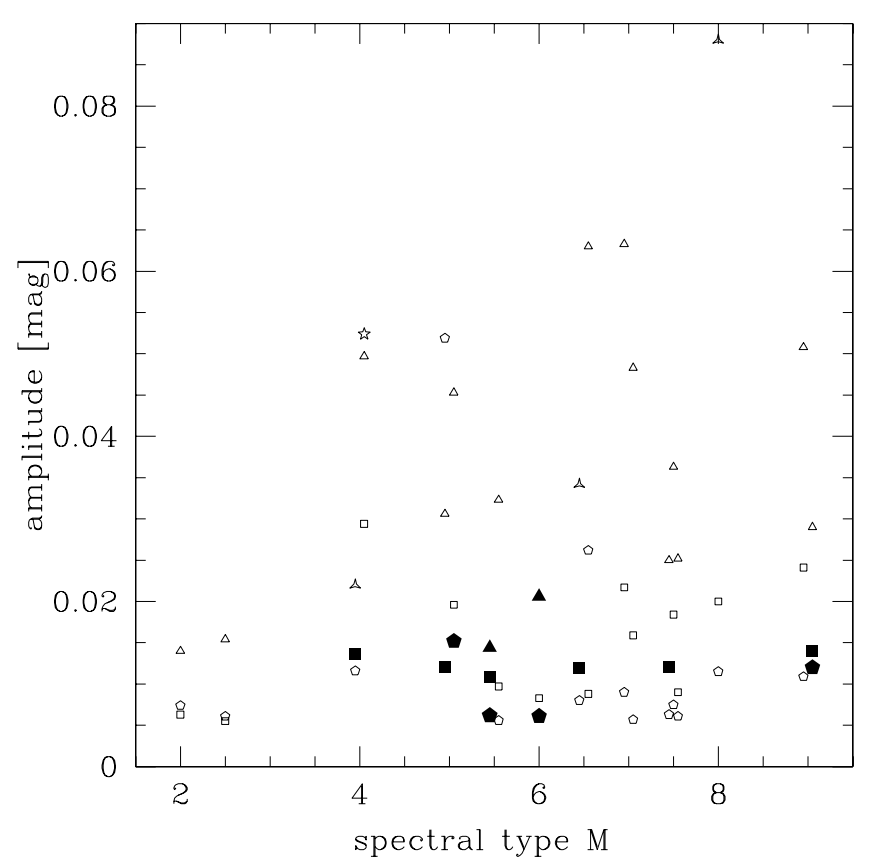

Fig. 14. Variability amplitudes (variable objects, solid markers) and upper detection limits (non-variable objects, open markers) of our data set versus M-spectral type. Channels $G, R$ and $I$ are represented by triangles, squares and pentagons, respectively. Starred symbols stand for rms amplitudes where the $\chi^{2}$ test gives a detection but variability is finally rejected.

with spectral type is apparent in either figure, although amplitudes of more than 0.02 mag have been found only at spectral types L0 to L3.

As most surveys have only been carried out in the $I$ band, we use just our $I$ band variability detections when comparing our results with other surveys. We then form three spectral type bins - early M (M0-M4, 4 objects), late M (M5-M9, 17 objects) and L (31 objects) - and calculate the fraction of variable objects in each. We thus arrive at fractions of $0.25 \pm 0.25$, $0.29 \pm 0.13$ and $0.48 \pm 0.12$ for these bins respectively (and $0.29 \pm 0.12$ for all $\mathrm{M}$ dwarfs together). Errors are derived assuming counting statistics. The difference between $\mathrm{M}$ and L type is about $1.5 \sigma$ which corresponds to a confidence level of about $86 \%$. Of particular interest is to compare only the L dwarfs from BJM01 with our M dwarfs because the analysis methods and sensitivity are very similar. We now find fractions of $0.21 \pm 0.11$ for the $19 \mathrm{M}$ dwarfs and $0.70 \pm 0.26$ for the $10 \mathrm{~L}$ dwarfs, which is a $2 \sigma$ difference. Both results suggest an increased frequency of occurrence of variability among $\mathrm{L}$ dwarfs. This is supported further by the fact that the present survey is more likely to detect variability in the $\mathrm{M}$ dwarfs on account of the multichannel observations.

On account of the incompleteness of further information on the targets as e.g. $\mathrm{H}_{\alpha}$ or $v \sin i$, we cannot properly test whether these quantities are significantly different between variable and non-variable objects. 


\section{Conclusions}

We have presented multiband data of $19 \mathrm{M}$ dwarfs of which seven show evidence for variability at a $99 \%$ confidence level in at least one of three channels. We performed relative photometry along with a careful error estimation in order to achieve the high photometric precision needed to detect low amplitude variability. Amplitudes (root-mean-square of the light curve) measured from 0.0055 to $0.014 \mathrm{mag}$ in $I$ and $R$ and from 0.014 to $0.034 \mathrm{mag}$ in G. For non-variable objects, upper limits to variability are estimated to lie between 0.006 and 0.05 mag.

Using the CLEAN algorithm to form the periodograms, convincing periodic variability was found in LHS370 and $2 \mathrm{M} 1707+64$ at periods of $5.9 \pm 2.0$ and $3.65 \pm 0.1 \mathrm{~h}$, respectively. We further claim these to be their rotation periods. Besides these two targets, three other objects show periodic variations in one channel: $2 \mathrm{M} 1344+77$ in $G$ at $12.5 \pm 2.0 \mathrm{~h}$; LHS2930 in $I$ at $13.2 \pm 1.9 \mathrm{~h} ; 2 \mathrm{M} 1714+30$ in $R$ at $6.9 \pm 0.15 \mathrm{~h}$. But here the evidence for periodicity is less convincing.

Various simulations were performed to determine both the period uncertainty and the probability of detecting an existing periodic signal for arbitrary time samplings. In this way we could confirm the high sensitivity of our data to the expected rotation periods of $\mathrm{M}$ dwarfs up to about $12 \mathrm{~h}$ (although early $\mathrm{M}$ dwarfs may have longer periods). With the help of synthetic spectra of Allard et al. (2001) the two favourite scenarios for variability in ultra cool dwarfs - magnetically induced spots and dust clouds - were studied in the cases of 2M1707+64 and LHS370. It turned out that the observed variability in the former object can be reproduced well by assuming an atmosphere of $2300 \mathrm{~K}, \log g=5.0$ and a $100 \mathrm{~K}$ cooler spot covering $7.5 \%$ of the visible star disk. Clear clouds on a dusty atmosphere or dusty clouds on a clear atmosphere can be ruled out. In contrast to this, the origin of variability in LHS370 remains unclear. The lack of detectable periods for most of our targets can be explained by absent or rapidly evolving surface features.

Combining the results of this and other works published in the recent years leads to evidence for an increased variability frequency in field L dwarfs when compared to field M-dwarfs. The fractions of variable objects are: early M type (M0-M4) $0.25 \pm 0.25$, late $M$ type (M5-M9.5) $0.29 \pm 0.13$ and $L$ type $0.48 \pm 0.12$. The number of objects in each bin is 4,17 and 31 , respectively. The difference between $M$ and $L$ type is about $1.5 \sigma$ which corresponds to an $86 \%$ confidence level. This is statistically not large but still hints at a more frequent variability in later spectral type. If we consider the homogeneous samples of this paper and BJM01, we find fractions of $0.21 \pm 0.11$ (19 M dwarfs) and $0.70 \pm 0.26$ (10 L dwarfs) which corresponds to a $2 \sigma$ difference.

Higher SNR data would allow us to put stronger constraints on variability sources, particularly if optical and infrared observations were combined. An increased number of monitored $\mathrm{M}$, late $\mathrm{L}$ and even $\mathrm{T}$ dwarfs could clarify the change of variability frequency with spectral type, and thus permit stronger conclusions. And while difficult to achieve because of the available telescope time, a denser and longer time sampling would make it possible to recover longer periods which are expected to be common among early $\mathrm{M}$ dwarfs.

Acknowledgements. We are very grateful to Roland Gredel, the director of the Calar Alto observatory, for a prompt and uncomplicated allocation of Directors Discretionary Time in 2005 and to the Calar Alto staff for obtaining these data and for their support during the 2002 and 2003 observing runs.

\section{References}

Allard, F., Hauschildt, P. H., Alexander, D. R., Tamanai, A., \& Schweitzer, A. 2001, ApJ, 556, 357

Bailer-Jones, C. A. L. 2002, A\&A, 389, 963

Bailer-Jones, C. A. L. 2004, A\&A, 419, 703

Bailer-Jones, C. A. L. 2005, Proc. 13th Cool Stars Workshop, ed. F.

Favata, G. A. J. Hussain, \& B. Battrick, ESA, SP-560, 429

Bailer-Jones, C. A. L., \& Lamm, M. 2003, MNRAS, 339, 477

Bailer-Jones, C. A. L., \& Mundt, R. 1999, A\&A, 348, 800

Bailer-Jones, C. A. L., \& Mundt, R. 2001, A\&A, 367, 218

Bessell, M. S. 1991, AJ, 101, 662

Clarke, F. J., Oppenheimer, B. R., \& Tinney, C. G. 2002, MNRAS, 335,1158

Clarke, F. J., Tinney, C. G., \& Hodgkin, S. T. 2003, MNRAS, 341, 239

Cruz, K. L., Reid, I. N., Liebert, J., Kirkpatrick, J. D., \& Lowrance, P. J. 2003, AJ, 126, 2421

Dahn, C. C., Harris, H. C., Vrba, F. J., et al. 2002, AJ, 124, 1170

Delfosse, X., Forveille, T., Perrier, C., \& Mayor, M. 1998, A\&A, 331, 581

Gelino, C. R., Marley, M. S., Holtzman, J. A., Ackerman, A. S., \& Lodders, K. 2002, ApJ, 577, 433

Gizis, J. E., Monet, D. G., Reid, I. N., et al. 2000, AJ, 120, 1085

Gorlova, N. I., Meyer, M. R., Rieke, G. H., \& Liebert, J. 2003, ApJ, 593, 1074

Henry, T. J., Subasavage, J. P., Brown, M. A., Beaulieu, T. D., \& Jao, W.-C. 2004, AJ, 128, 2460

Kirkpatrick, J. D., McGraw, J. T., Hess, T. R., Liebert, J., \& McCarthy, D. W. J. 1994, ApJS, 94, 749

Koen, C. 2005, MNRAS, 357, 1151

Lamm, M. H., Bailer-Jones, C. A. L., Mundt, R., Herbst, W., \& Scholz, A. 2004, A\&A, 417, 557

Leggett, S. K., Allard, F., Dahn, C. C., et al. 2000, ApJ, 535, 965

Martín, E. L., Delfosse, X., Basri, G., et al. 1999, AJ, 118, 2466

Martín, E. L., Zapatero Osorio, M. R., \& Lehto, H. J. 2001, ApJ, 557, 822

Mohanty, S., \& Basri, G. 2003, ApJ, 583, 451

Mohanty, S., Basri, G., Shu, F., Allard, F., \& Chabrier, G. 2002, ApJ, 571,469

Reid, I. N., Kirkpatrick, J. D., Liebert, J., et al. 2002, AJ, 124, 519

Roberts, D. H. 1987, AJ, 93, 968

Rockenfeller, B. 2005, Diploma thesis, University of Heidelberg, Germany

http://www.mpia.de/homes/calj/MV_survey.html

Rockenfeller, B., Bailer-Jones, C. A. L., Mundt, R., \& Ibrahimov, M. 2006, MNRAS, in press

Scholz, A., \& Eislöffel, J. 2004a, A\&A, 421, 259

Scholz, A., \& Eislöffel, J. 2004b, A\&A, 419, 249

West, A. A., Hawley, S. L., Walkowicz, L. M., et al. 2004, AJ, 128, 426 